\title{
Der Erfolg der „Dummheit”
}

\section{Deep Blues Sieg über den Schachweltmeister Garri Kasparov und der Streit über seine Bedeutung für die Künstliche Intelligenz-Forschung}

\author{
Martina Heßler
}

The Triumph of "Stupidity". Deep Blue's Victory over Garri Kasparov. The Controversy about its Impact on Artficial Intelligence Research

The competition between the chess computer Deep Blue and the former chess world champion Garri Kasparov in 1997 was a spectacle staged for the media. However, the chess game, like other games, was also a test field for artificial intelligence research. On the one hand Deep Blue's victory was called a "milestone" for Al research, on the other hand, a dead end, since the superiority of the chess computer was based on pure computing power and had nothing to do with "real" Al.

The article questions the premises of these different interpretations and maps Deep Blue and its way of playing chess into the history of Al. This also requires an analysis of the underlying concepts of thinking. Finally, the essay calls for assuming different "ways of thinking" for man and computer. Instead of fundamental discussions of concepts of thinking, we should ask about the consequences of the human-machine division of labor.

Keywords: History of artificial intelligence research, Deep Blue, Chess computer, Thinking

Der Wettkampf zwischen dem Schachcomputer Deep Blue und dem damaligen Schachweltmeister Garri Kasparov im Jahr 1997 war ein medienwirksam inszeniertes Spektakel. Darüber hinaus war das Schachspiel aber, wie auch andere Spiele, ein Testfeld der Künstlichen Intelligenz-Forschung. Der Sieg Deep Blues wurde einerseits als "Meilenstein" der KI-Forschung bezeichnet, andererseits als "Sackgasse”, da die Überlegenheit des Schachcomputers auf reiner Rechengewalt beruhe und nichts mit "wirklicher KI" zu tun habe.

Der Beitrag fragt nach den Prämissen dieser unterschiedlichen Interpretationen und ordnet Deep Blue und seine Weise des Schachspielens in die Geschichte der KI ein. Dafür ist auch die Analyse der zugrundeliegenden Begriffe von Denken notwendig. Schließlich plädiert der Aufsatz dafür, von unterschiedlichen Denkweisen von Mensch und Computer auszugehen und statt der Grundsatzdiskussionen um den Begriff des Denkens nach den Konsequenzen der menschlich-maschinellen Arbeitsteilung zu fragen.

Schlüsse/wörter: Geschichte der Künstlichen Intelligenz-Forschung, Deep Blue, Schachcomputer, Denken

Der schachspielende IBM-Computer Deep Blue gehört zweifellos zu den bekanntesten Maschinen des 20. Jahrhunderts. Sein Sieg gegen den damals amtierenden und langjährigen Schachweltmeister Garri Kasparov im Jahr 1997 war ein weithin wahrgenommenes und debattiertes Ereignis und zugleich ein gekonnt inszenierter Werbecoup der Firma IBM. Der Wettkampf wurde in populären Medien aufgenommen und beispielsweise in einem als 
Thriller inszenierten Dokumentarfilm mit dem Titel Game Over verarbeitet. Schachspielende Computer waren auch in anderen Filmen Hauptfiguren, bis hin zu HAL in Stanley Kubricks Film Odyssee 2001. ${ }^{1}$ Der Sieg Deep Blues entfachte zudem ein ungeheures Medienecho. Dies ist wenig überraschend, schließlich galt Schachspielen als genuin menschliche Leistung und als Ausweis von Intelligenz. Das klassische Narrativ „Kampf Mensch gegen Maschine" ließ sich hier besonders leicht und spektakulär inszenieren. Bereits vor den Spielen gegen Garri Kasparov hatte es eine Reihe öffentlicher Wettkämpfe „Mensch gegen Computer" gegeben. So fand in den 1970er Jahren eine Fülle von Spielen zwischen Mensch und Computer statt (vgl. Levy 1976: 113-129); in der Schweiz wurden Meisterschaften zwischen Mensch und Computer abgehalten. ${ }^{2}$ Im Zweiten Deutschen Fernsehen war im Jahr 1979 ein Wettkampf zwischen dem damals als führend geltenden Schachprogramm Chess 4.8 und dem Internationalen Meister David Levy zu sehen. ${ }^{3}$ Levy hatte überdies im Jahr 1968 eine Wette abgeschlossen, dass er innerhalb der nächsten zehn Jahre nicht gegen einen Computer verlieren würde - eine Wette, die er gewann. Und auch 1979 ließ er sich nicht vom Schachprogramm besiegen (Coy 1993: 212 f.). ${ }^{4}$ Gleichfalls im Jahr 1979 fand ein Simultanspiel eines Schachcomputers gegen neun Schachspieler in Hamburg statt, veranstaltet vom Magazin Der Spiegel. ${ }^{5}$

Der Kulturwissenschaftler Hartmut Böhme hatte den Wettkampf zwischen Kasparov und Deep Blue als Albernheit bezeichnet (Böhme 2009: 68). „Wettkämpfe" dieser Art, wie es sie auch jenseits des Schachs vielfach gab und gibt, sind jedoch nicht schlichtweg albern. Es handelt sich um ein eigenes Genre, das innerhalb der Künstlichen Intelligenz (KI)-Forschung verschiedene Funktionen erfüllt. Zum einen eine demonstrierende Funktion, nämlich als öffentlichkeitswirksame Werbeaktionen, die Aufmerksamkeit sichern, Erfolge demonstrieren und letztlich auch Fördergelder fließen lassen sollen. Zum zweiten sind die Spiele Testfelder, an denen Paradigmen der KI-Forschung erprobt werden. Ihre Bedeutung reduziert sich daher nicht auf das Eventhafte, Spektakuläre und Werbewirksame; sie sind zugleich wichtiger Bestandteil der Forschung. Zum dritten machen sie die Ergebnisse der KI-Forschung in einer breiten Öffentlichkeit sichtbar und führen zu intensiven Debatten.

Die öffentliche Diskussion um das Spektakel um Deep Blue drehte sich vorrangig um die Frage der Überlegenheit von Maschinen über Menschen. Der Schachcomputer weckte die klassischen Ängste vor der Dominanz der Maschine, vor menschlicher Ohnmacht sowie vor der Entthronung des Menschen als einzigem denkenden Wesen.

David Jay Bolter sprach im Kontext des Computers generell von einer "defining technology“ (Bolter 1984). Er bezeichnete damit Technologien, die das Verhältnis von Mensch und Natur, wie er 1984 schrieb, sowie die 
Selbstdefinitionen der Menschen verändern (Bolter 1984: 10). Er fragte, metaphorisch gesprochen, wie der Mensch in den Computer kam, um damit, in allerdings tendenziell kulturkritischer Manier, „a change in the way men and women in the electronic age think about themselves and the world around them" zu beschreiben (Bolter 1984: 4). Gerade die Künstliche Intelligenz-Forschung mit ihrem Bestreben menschliche Intelligenz zu imitieren, berührt fundamentale Fragen der Bestimmung des Menschseins. ${ }^{6}$

Im Folgenden wird dieser Aspekt allerdings nicht im Zentrum stehen. Vielmehr wird der Wettkampf zwischen Deep Blue und Kasparov in die Geschichte der KI-Forschung eingeordnet. Dabei verfolgt der Beitrag zwei zentrale Anliegen.

Erstens soll das meist zu findende Standardnarrativ der KI-Geschichte hinterfragt und differenziert werden. Dieses beschreibt üblicherweise einen Wechsel von einem kognitivistischen Paradigma in der Frühphase der KI-Forschung hin zum Konnektionismus/neuronalen Netzen und „verhaltensbasierten“ oder „embodied-intelligence“-Ansätzen seit den 1980er Jahren. ${ }^{7}$ Im Kontext dieser Lesart der Geschichte der KI wird Deep Blue als eine Sackgasse interpretiert (z. B. Ensmenger 2012: 23), als eine Spielerei, die die KI-Forschung nicht vorangebracht habe. Allerdings wurde der Sieg Deep Blues gleichzeitig - wie im übrigen Watsons Sieg in einer amerikanischen Spielshow im Jahr 2011 - als ein großartiger Erfolg der KI-Forschung und als Meilenstein der Computergeschichte bezeichnet (Schaeffer \& van den Herik 2002: 3; Newborn 2003). Dies wird im Folgenden ausführlicher dargestellt und die unterschiedlichen Argumente auf ihre Prämissen hin analysiert, um zu einem neuen Blick auf die KI-Geschichte zu gelangen. Dabei argumentiert der Beitrag auch, dass die zumeist zu findende klare Trennung von theoretischem Interesse, von Grundlagenforschung einerseits und Anwendung andererseits, also von starker und schwacher KI, häufig zu strikt gezogen wird. ${ }^{8}$ Gerade das Schachspiel war, so die im Folgenden zu entwickelnde These, ein Forschungsfeld der KI, das gleichermaßen fundamentalen Einsichten in das menschliche Gehirn sowie der praktischen Anwendung dienen sollte. Hier offenbart sich zugleich der Pragmatismus vieler KI-Forscher, die, wie Noam Chomsky im Hinblick auf Forschung generell formulierte, „tend to study what you know how to study" (Chomsky 2012).

Schließlich entzündeten sich an Deep Blue, wie erneut am Sieg des Computers Watson in einer amerikanischen Spielshow 2011, gesellschaftliche Debatten, ob Maschinen denken können und was Denken sei. Dabei wurden die Standardargumente, die seit Beginn der KI-Forschung zu finden sind, in scharfen Kontroversen ausgetauscht. Das zweite zentrale Anliegen des Beitrags ist es, diese Debatte, die in den Kern der KI-Geschichte führt, 
zu untersuchen. Dabei zeigt sich die Historizität und Polyvalenz der Begriffe. Einerseits steht der Begriff des Denkens innerhalb der frühen KI in einer langen Tradition des logischen und kalkulierenden Denkens, während Kritiker dies als Verkürzung deuteten. Zudem drehte sich die Debatte immer wieder darum, ob Denken den Menschen vorbehalten oder ob das, was der Computer mache, auch als denken zu bezeichnen sei. Hier zeigt sich eine anthropozentrische Verteidigung des menschlichen Denkens versus pragmatischer Ingenieurs- und Programmiererlogik, für die das Ergebnis zählt: etwa, dass ein menschlicher Schachweltmeister besiegt wird.

Der Aufsatz zeichnet diese Debatte am Beispiel von Deep Blue, Watson und Alpha Go nach. In dieser Debatte ist eine strikte, kategoriale Trennung menschlicher und maschineller Intelligenz zu beobachten, die einer Grundlagendiskussion um Begrifflichkeiten gleicht, die aber, so die These, die viel entscheidenderen Fragen nach den Konsequenzen einer maschinellen Intelligenz verfehlt, wie im letzten Abschnitt dargelegt werden wird.

Im Folgenden werden zuerst die spektakuläre Story um Deep Blue sowie die Geschichte des Computerschachs und dessen Rolle in der frühen KIForschung skizziert. Hier wird der Begriff des Denkens, wie er in der frühen KI-Forschung dominierte, historisiert und dessen Kompatibilität mit der Computerlogik aufgezeigt. Anschließend gilt es, die Methode, mit der Deep Blue Schach spielte, die sogenannte Brute-Force-Strategie, nachzuzeichnen, um Deep Blue in die Geschichte der KI-Forschung einordnen zu können. Es folgt der Blick auf die im Anschluss an Deep Blue erhitzt geführte Debatte, ob der Sieg über den Schachweltmeister etwas mit Denken zu tun habe und ob es sich um "wirkliche KI" handele. Abschließend wird die Frage nach Erfolg oder Sackgasse, nicht zuletzt mit einem Blick auf den IBM-Computer Watson und Alpha-Go, erneut gestellt und dabei für neue Perspektiven auf die Geschichte der KI-Forschung argumentiert.

$\mathrm{Zu}$ Deep Blue liegt bislang keine historische Studie vor. Allerdings war der siegreiche Schachcomputer vielfach Thema verschiedenster Veröffentlichungen. Teils handelt es sich um populärwissenschaftliche Bücher, häufig von Schachspielern verfasst. Weiter schilderten beteiligte Akteure, wie der Vorsitzende des Computer Chess Committees for the Association of Computing Machines, Monty Newborn, oder einer der Entwickler von Deep Blue, Feng-Hsiung Hsu, ihre Perspektive der Geschichte. Gleiches gilt für die Geschichte des Computerschachs, die kaum aufgearbeitet ist. Einen wichtigen Beitrag lieferte der Computerhistoriker Nathan Ensmenger mit einem Aufsatz, auf den noch zurückzukommen sein wird (Ensmenger 2012). In den 1990er Jahren beschäftigte sich auch Wolfgang Coy in einem Aufsatz mit Computerschach (Coy 1993). Des Weiteren finden sich wiederum Darstellungen von computer scientists sowie allgemeine Abhandlungen zur Geschichte des Schachspiels, in denen auch das Computerschach Berück- 
sichtigung findet, sowie populärwissenschaftliche Publikationen zum Thema Computerschach, die insbesondere in den 1970er und 1980er Jahren entstanden sind. ${ }^{9}$ Genau wie die zeitgenössische Debatte um Deep Blue und um die Frage des maschinellen Denkens werden diese Publikationen hier als Quellen herangezogen. ${ }^{10}$

\section{Die Story: Deep Blues Sieg über den Schachweltmeister}

Der IBM Computer Deep Blue war der erste Schachcomputer, der 1996 einen Schachweltmeister in einem Spiel und im Jahr 1997 schließlich in einem Turnier schlug. 1996 hatte Kasparov das Turnier gegen Deep Blue in Philadelphia noch gewonnen, auch wenn er ein Spiel des Matches verloren hatte. Das Turnier 1997 war die Revanche, für die Deep Blue gegenüber dem Vorjahresturnier erheblich verbessert worden war (Campbell et al. 2002: 57). Wie einer der Entwickler formulierte, habe es sich nicht mehr um dieselbe Maschine gehandelt. Am 11. Mai 1997 gewann dieser überarbeitete Deep Blue schließlich gegen den amtierenden Schachweltmeister in einem Sechs-Spiele-Match. Zwei Spiele gewann Deep Blue, eines Kasparov, und drei verliefen unentschieden. Das letzte von sechs Spielen hatte der Computer nach nur 19 Zügen für sich entschieden (Goodman \& Keene 1997: 100-106). ${ }^{11}$

Das Match dauerte insgesamt acht Tage und entfachte ein massives Medien-Echo. Es hatte vor laufenden Kameras in einem Fernsehstudio in New York stattgefunden. Zudem wurde es im Internet übertragen und noch 2001 als das „größte Internetereignis aller Zeiten“ tituliert. Die Veranstalter verzeichneten stundenweise über 22 Millionen Zugriffe auf die Homepage des Turniers; entsprechend brach die Seite mehrfach zusammen. ${ }^{12}$ In den Medien erschienen zahlreiche Artikel, die das Ereignis in seiner Bedeutung für die Menschheit, die KI-Forschung und für das Schachspiel interpretierten und insbesondere den Kampf zwischen Mensch und Maschine zum Thema machten, um dabei an uralte Ängste der Menschen vor der Überlegenheit der Maschine zu rühren. ${ }^{13}$

Der Sieg über Kasparov war insofern besonders spektakulär, da erstens die KI-Forschung seit Jahrzehnten an Schachcomputern arbeitete und Schach als eines der zentralen Paradigmen der KI-Forschung galt. Wie Monty Newborn etwas pathetisch zusammenfasste, war Deep Blue das Ergebnis der Arbeit von Tausenden Forschern, die in den späten 1950er Jahren begonnen hatten, an Schachcomputern zu arbeiten (Newborn 2000: 27). 1957 hatte Herbert Simon prognostiziert, dass in zehn Jahren ein Computer Schachweltmeister sein würde (Bruns 2000: 313). Einen Schachwelt- 
meister zu besiegen, war ein prestigereiches Ziel der KI-Forschung, das schließlich 1997 erreicht wurde.

Zweitens war der Sieg von Deep Blue deshalb spektakulär, weil gerade Garri Kasparov als eine besondere Herausforderung galt. Er war ein Ausnahmespieler, der als unbesiegbar bezeichnet wurde. Er hatte von 1986 bis 2005 den ersten Rang der Weltrangliste der Schachprofis inne (Ensmenger 2012: 22), und damit bis heute weitaus länger als alle anderen professionellen Schachspieler.

Der Niederlage Kasparovs folgte eine Reihe weiterer Spiele zwischen Großmeistern und Schachcomputern. Kasparov hatte sogleich eine Revanche gefordert, doch IBM lehnte dies ab (Hsu 2002: 261). Deep Blue wurde eingemottet und später an das Smithonian Museum in Washington überführt. Es fanden jedoch weitere Spiele Kasparovs und auch Vladimir Kramniks, der Kasparov als Schachweltmeister im Jahr 2000 abgelöst hatte, gegen Schachcomputer statt, so in den Jahren 2002, 2003 und 2006. Meist kam es dabei zu einem Remis, sehr selten siegte der menschliche Spieler. 2006 verlor Kramnik deutlich gegen Deep Fritz. Das Turnier 2006 gilt als Ende der Wettkämpfe zwischen Schachcomputern und Menschen und etablierte gleichzeitig die Erkenntnis, dass der Computer dem Menschen im Schachspielen nun endgültig und uneinholbar überlegen sei.

\section{Eine kurze Skizze des Computerschachs ${ }^{14}$ und die Tradition des logisch-formalen Denkens}

Die Idee, das Schachspiel an Maschinen zu delegieren, findet sich bereits im 18. Jahrhundert. Zu erinnern ist an Wolfgang von Kempelens „Schachtürken“, den er 1769 präsentierte, der sich allerdings gerade nicht als eine denkende Maschine, sondern als eine Täuschung erwies, da im Inneren des Automaten ein Schachmeister versteckt war. Gleichwohl tourte Kempelens Automat bis in das späte 19. Jahrhundert als vielbeachtetes Spektakel durch Europa. Edgar Allen Poe kommentierte den Automaten in einem bekannten Essay (vgl. Coy 1993). Charles Babbage hatte darüber nachgedacht, dass der von ihm entwickelte Rechenautomat Analytical Engine Schach spielen könne (Bell 1978: 12 f.). Ende des 19. Jahrhunderts hatte der spanische Elektromechaniker Leonardo Torres y Quevedo eine Maschine entwickelt, die immerhin ein bescheidenes Endspiel beherrschte (Pfleger \& Weiner 1986: 15 f.; Shannon 1950).

Computerschach entstand jedoch erst mit der KI-Forschung in der Mitte des 20. Jahrhunderts. Die Geschichte des Computerschachs ist von hoher Relevanz für die Geschichte der KI-Forschung (Franchi \& Güzeldere 2005: 
46-58; Ensmenger 2012). Hier verband sich die Idee der Delegierung von Schachspielen, Rechnen oder Denken auf Maschinen mit der Logik des Computers. Um das Computerschach und Deep Blue historisch einzuordnen, ist ein Blick zurück auf die Geschichte des Begriffs des Denkens notwendig.

Wie Barbara Becker konstatiert hatte, waren „die meisten neuzeitlichen Theorien und Entwürfe über den menschlichen Geist theoretische Abhandlungen“, bis sich „mit der Erfindung und Verbreitung der Computertechnologie in den 50er Jahren plötzlich eine neue Forschungsperspektive“ eröffnete (Becker 1992: 94). Zu diesem Zeitpunkt wurde Schach ein zentrales Paradigma der sich konstituierenden KI-Forschung. Schach galt als die „Drosophila“ des Forschungsfeldes (Franchi \& Güzeldere 2005: 47; Ensmenger 2012: $5 \mathrm{f}$. $)^{15}$ also, in Analogie zur Genetik, als ein relativ einfaches Modell, an dem weitreichende Theorien, hier über den menschlichen Verstand, das Gehirn und die Intelligenz, entwickelt werden könnten.

Die Erforschung von Spielen generell, nicht nur von Schach, gilt als „longest running experiment in computing-science history“. Spiele, insbesondere Schach, dienten als „experimental test beds for many areas of artificial intelligence" (Schaeffer \& van den Herik 2002: 6). Wie Claude Shannon in seinem Aufsatz „Programming a Computer for Playing Chess“ schrieb: „Although perhaps of no practical importance, the question is of theoretical interest, and it is hoped that a satisfactory solution of this problem will act as a wedge in attacking other problems of a similar nature and of greater significance“ (Shannon 1950: 256).

Die bedeutende Rolle des Schachs hierbei erklärt sich erstens aus dem Image des Schachspiels als Spiel, das ein Indikator von Intelligenz sei. Es war etwas, was nur Menschen taten (nicht Tiere), etwas, wobei man denken musste. Sofern ein Computer Schach spielen und gar menschliche Spieler schlagen könne, sei er intelligent, so die Annahme vieler KI-Forscher. Shannon schrieb: „[C]hess is generally considered to require 'thinking' for skilful play" (Shannon 1950: 257). Newell, Shaw and Simon formulierten: "Chess is the intellectual game par excellence. [...] If one could device a successful chess machine, one would seem to have penetrated to the core of human intellectual endeavour" (Newell et al. 1958: 320).

Zweitens war es ein zentrales Ziel der frühen KI-Forschung, die Funktionsweise des Gehirns mittels der Analogie zum Computer besser zu verstehen. Dies ist wiederum nur vor dem Hintergrund des Konzeptes, was Denken sei, nachzuvollziehen. Denn die Grundannahme der frühen KIForschung - also des als kognitivistisch oder rationalistisch bezeichneten Paradigmas - war, dass Denken Informationsverarbeitung sei, auf der Befolgung reproduzierbarer Regeln basiere und damit formalisierbar ist (Dittmann 2015: 241; Zimmerli \& Wolf 1994: 14). Diese Definition des 
Denkens schloss an eine lange Tradition an, das Denken formal-logisch zu interpretieren. „Die Meinung, wie weit die Geschichte der KI zurückreiche“, sei daher geteilt, wie Zimmerli und Wolf konstatierten: „Nicht nur beginnen fast alle Darstellungen mit antiken Vorläufern der KI-Diskussion, sondern es wird auch in der Neuzeit der Anfang bald mit Descartes, bald mit Leibniz, bald mit Babbage, bald mit Turing, bald mit McCarthy gemacht" (Zimmerli \& Wolf 1994: 7). Descartes hatte in der mathesis universalis formales Schlussfolgern als eine Art von Rechnen interpretiert, Hobbes konstatierte, dass Denken nichts anderes als Rechnen sei (ebd.: 10; hier auch Haugeland 1987: 19-38). Insbesondere mit Leibniz „avanciert Berechenbarkeit zum Vernunftkriterium“ (Zimmerli \& Wolf 1994: 10; vgl. auch Krämer 1988).

Nicht zufällig dachte also bereits Babbage bei der Entwicklung seiner Rechenmaschine auch darüber nach, ob diese Schach spielen könne. Das Schachspiel mit seinen klaren Regeln und seiner logischen Struktur entsprach dem Konzept, was Denken sei, wie es seit der Frühen Neuzeit im Sinne einer Formalisierung und Kalkülisierung formuliert wurde. Schach wurde daher nicht zufällig zur „Drosophila“ der KI-Forschung. Vielmehr entsprach es den historisch wirkmächtigen Konzepten: ${ }^{16}$ Wenn Denken formal-logisch funktioniert, dann kann man es, so die Annahme, an Maschinen delegieren. Im Hinblick auf Schach hatte Shannon formuliert: "A solution of this problem will force us either to admit the possibilities of a mechanized thinking or to further restrict our concept of thinking“ (Shannon 1950: 257).

Drittens war Schach für die Forschungen der frühen KI zugleich aus pragmatischen Gründen geeignet, wie Shannon in seinem Aufsatz von 1950 ausführte. Schach eigne sich, weil „(1) the problem is sharply defined both in allowed operations (the moves) and in the ultimate goal (checkmate); (2) it is neither so simple as to be trivial nor too difficult for satisfactory solution“. Und schließlich: „[... ] the discrete structure of chess fits well into the digital nature of modern computers" (Shannon 1950: 257).

Die weiter unten referierte Debatte um das Denken kann nur vor dem Hintergrund dieser Tradition der Bestimmung des Denkens als formallogisch verstanden werden. Gerade die Kritiker der KI-Forschung wie der Philosoph Hubert Dreyfus arbeiteten sich an einem solchen Verständnis des Denkens ab. Dieser Denkbegriff entsprach jedoch dem Schachspielen genauso wie der Logik des Computers. Gewissermaßen verband sich hier in gleichsam zirkulärer Weise eine historische Tradition des Verständnisses dessen, was Denken sei mit der Logik des Computers sowie mit der Regelhaftigkeit und formalen Struktur des Schachspiels. Für ein Grundverständnis des so konzipierten Denkens und seiner Übertragung auf Maschinen schien dies jedoch vielversprechend. 
Entsprechend prominent war die Rolle des Computerschachs in der KIForschung: „Hundreds of academic papers have been written about computer chess, thousands of working chess programs have been developed, and millions of computer chess matches have been played" (Ensmenger 2012: 6). Alan Turing hatte bereits 1946 über eine Schachmaschine nachgedacht und 1953 auf dem Papier ein Schachprogramm entworfen (Turing 1987). Auch Konrad Zuse sowie Shannon, McCarthy, Newell, Simon und andere frühe KI-Forscher befassten sich mit Schach (vgl. Levy \& Newborn 1991: 24-38).

In der Frühphase realisierten allerdings wenige die Probleme und die Zeit, die es dauern würde, bis ein Computer einen menschlichen Schachweltmeister schlagen könne. Vielmehr fanden sich viele optimistische Vorhersagen (Schaeffer \& van den Herik 2002: 1), so auch die von Shannon aus dem Jahr 1957, dass innerhalb der nächsten zehn Jahre ein Schachcomputer Weltmeister werden würde. In den 1950er Jahren hatte John von Neumann ein Programm für ein $6 \times 6$ Schachbrett entwickelt. 1958 schrieben Alex Bernstein und Michael de V. Roberts ein Schachprogramm für den IBM-704-Computer, das in der Zeitschrift Scientific American veröffentlich wurde (Newell et al. 1958). 1974 hatte die erste SchachcomputerWeltmeisterschaft in Stockholm, 1980 die erste Mikrocomputer-Weltmeisterschaft stattgefunden (Pfleger \& Weiner 1986: 22).

Die anfänglichen Erfolge in den 1950er Jahren lösten Euphorie und hohe Erwartungen aus. Doch während Laienspieler schnell besiegt wurden, verloren Computer lange Zeit gegen Schachmeister. Erst 1988 besiegte der Computer Deep Thought einen Großmeister in einem Spiel (Campbell et al. 2002: 58). Im Jahr 1997 gelang dann schließlich der Sieg des IBMComputers Deep Blue gegen Kasparov.

Bei IBM hatte seit 1989 ein Team an einem Schachcomputer gearbeitet. Die Forschungen, die schließlich zu Deep Blue führten, waren allerdings bereits Mitte der 1980er Jahre an der Carnegie Mellon University in Pittsburgh begonnen worden. Eine Gruppe von Doktoranden, darunter Murray Campbell und Feng-Hsiung Hsu, hatte angeregt durch die Arbeiten von Ken Thompson begonnen, Chips für das Computerschachspiel zu entwickeln. (Goodman \& Keene 1997: 11; vgl. ausführlich: Hsu 2002). Hsu arbeitete an der Hardware, während sich Campbell der Software widmete. Ein Ergebnis ihrer Forschungen war der gerade schon erwähnte Deep Thought, der 1988 den dänischen Großmeister Bent Larsen besiegte. Deep Thought hatte 1989 auch gegen Kasparov gespielt, aber deutlich verloren. Ende der 1980er Jahre wurden Campbell und Hsu von IBM eingestellt, um am Computerschach zu arbeiten. An der Entwicklung von Deep Blue waren auch Schachmeister beteiligt, insbesondere der amerikanische Meister Joel Benjamin, bei dem Deep Blue in die "Schachschule“ ging. Insgesamt 
hatte die Entwicklung Deep Blues zwölf Jahre gedauert. Gerade das Buch von $\mathrm{Hsu}$, in dem er die Entwicklung beschreibt, macht deutlich, welche immense Rolle die Hardware und die Geschwindigkeit spielten. Nicht zuletzt wird in seinen Beschreibungen auch offensichtlich, welch immense Anstrengung und wie viel Zeit, inklusive der Rückschläge, die Entwicklung Deep Blues kostete.

\section{Die A- und B-Strategie: Wie spielen Computer Schach?}

Claude Shannon hatte im Jahr 1950 einen wegweisenden, vielbeachteten und vielzitierten Artikel über die Möglichkeiten und Ansätze der Schachprogrammierung verfasst (Shannon 1950). Dabei unterschied er zwei Weisen, nämlich die A- und die B-Strategie. Die A-Strategie setzt auf die Errechnung aller überhaupt möglichen Züge bis zu einer bestimmten Tiefe. Der Rechner durchsucht Entscheidungsbäume. Diese Strategie erfordert immense Rechenleistungen, da auch völlig sinnlose Züge errechnet werden. Es handelt sich um die sogenannte Brute-Force-Methode. Im Jahr 1950 konstatierte Shannon allerdings noch:

Unfortunately a machine operating according to the type A strategy would be both slow and a weak player. It would be slow since even if each position was evaluated in one microsecond (very optimistic) there are about $10^{9}$ evaluations to be made after three moves (for each side). Thus, more than 16 minutes would be required for a move, or 10 hours for its half of a 40-move game. (Shannon 1950: 269)

Die Notwendigkeit und Bedeutung hoher Rechenleistung wird in diesem Zitat deutlich, genauso wie wenig absehbar es schien, dass diese fast 50 Jahre später zur Verfügung stand.

Die B-Strategie setzt darauf, sinnlose Züge zuerst zu erkennen und statt des Durchrechnens aller Züge mit Bewertungen der Situation und der Privilegierung bestimmter Möglichkeiten zu beginnen: „Select the variations to be explored by some process so that the machine does not waste it's time in totally pointless variations" (Shannon 1950: 270). Diese auch "selektiv“ genannte Strategie galt dem menschlichen Denken ähnlicher (Newell et al. 1958). ${ }^{17}$ Da sie jedoch weitaus schwieriger umzusetzen war, konzentrierten sich die Bemühungen bald auf die A-Strategie (Pfleger \& Weiner 1986: 17). Dies ist in insofern wichtig, als, wie Nathan Ensmenger in seinem Aufsatz aus dem Jahr 2012 aufzeigte, die Entscheidung für die A-Logik den Gang der KI-Forschung prägte und zu einem dominanten Ansatz wurde. Vie- 
len gilt dies als „Sündenfall“18 der KI-Forschung, da dies in eine Sackgasse geführt habe.

Die Methode wurde insbesondere mit der steigenden Computerkapazität in den 1970er und 1980er Jahren weiterentwickelt. Es wurde deutlich, dass eine klare Korrelation zwischen der Suchgeschwindigkeit, der Suchtiefe und dem Erfolg des Programms lag. Je höher die Rechenleistung des Computers, desto höher seine Gewinnchancen. Man arbeitete daher an der Entwicklung von schnellen Suchmaschinen (Schaeffer \& van den Herik 2002: 2). Die Forschung zu Computerspielen konzentrierte sich also deutlich auf die Brute-Force-Methode, obgleich es immer Zweifel an dieser Forschungsrichtung gab. ${ }^{19}$ Nach langsamen Entwicklungen sowie vielen Versuchen und Turnieren in den 1980er Jahren wurden schließlich in den 1990er Jahren zunehmend Erfolge in Spielen gegen Großmeister erzielt (vgl. oben).

Deep Blue basierte auf einem Suchalgorithmus, einer Bewertungsfunktion der Züge und einer riesigen Datenbasis mit gespielten Partien. Der Computer war 1997 in der Lage 200 Millionen Stellungen in einer Sekunde $\mathrm{zu}$ „prüfen“ und sich dann für einen Zug zu entscheiden. Zum Erfolg verhalf Deep Blue vor allem auch die Bewertungsfunktion, die gemeinsam mit den Schachmeistern Joel Benjamin und Miguel Illesacs entwickelt worden war sowie die Datenbanken über Eröffnungen und Endspiele (Newborn 2000: 27; Campell et al. 2002: 79).

\section{Deep Blues umstrittene Bedeutung für die Geschichte der KI- Forschung}

Innerhalb der Geschichtsschreibung zur KI-Forschung sowie auch von ihren Kritikern wurde vielfach und recht einhellig betont, die Entscheidung für die und die Dominanz der sogenannten A-Strategie, also der BruteForce-Methode, habe die KI von ihrem eigentlichen Ziel, das menschliche Denken zu verstehen, abgebracht. Nathan Ensmenger hatte dies explizit auf die Bedeutung des Schachspiels innerhalb der KI-Forschung zurückgeführt. Gerade die Konzentration auf Schach habe in eine theoretische Sackgasse geführt, insofern sei aus all den aufwändigen Forschungen keinerlei neue theoretische Erkenntnisse über die Funktionsweise des menschlichen Gehirns hervorgegangen, womit das Ziel der KI-Forschung verfehlt worden sei. Ensmengers Einschätzung entspricht den Narrativen der Geschichtsschreibung, in der betont wird, dass das kognitivistische Paradigma, die nach Haugeland sogenannte „Good Old Fashion AI“ (GOFAI) (Haugeland 1987: 96), in eine Sackgasse geführt habe. ${ }^{20}$ Dies geht mit einem inzwi- 
schen dominant gewordenen Narrativ einher, das für die 1980er Jahre von einem „AI Winter“ (Nocks 2008: 82) spricht, da die ausbleibenden Erfolge der KI-Forschung dazu geführt hatten, dass Fördergelder seitens des Staates reduziert worden waren. Damit einhergegangen war demnach ein Paradigmenwechsel weg vom kognitivistischen Paradigma hin zum Konnektionismus, den neuronalen Netzen sowie zu einem verhaltensbasierten und „embodied“-Ansatz (vgl. etwa die Überblicke Nocks 2008; Lenzen 2002). Oder wie John Haugeland es formuliert hatte, die erste Phase der KI-Forschung galt als beendet (Haugeland 1987: 96).

So liest sich Deep Blues Sieg aus zweifacher Perspektive als eine Sackgasse. Erstens war Deep Blue aus Sicht der sogenannten starken KI, die darauf abzielt, Erkenntnisse über die Funktionsweise des menschlichen Gehirns zu erhalten und das menschliche Denken zu verstehen, ein Misserfolg. Die ungeheure Rechengewalt und Computerpower, die Methoden, mit denen Deep Blue zum Ziel kam, gleichen keineswegs der menschlichen Intelligenz. Es konnten keine Erkenntnisse über die Funktionsweise des menschlichen Gehirns gewonnen werden. Zweitens basiert Deep Blue auf einem formal-logischen Ansatz, auf einer Programmierung und strukturierten Datenbanken. Dieser die frühe KI-Forschung dominierende Ansatz gilt inzwischen als überholt. Er wurde vom sogenannten Konnektionismus, den neuronalen Netzen abgelöst. Deep Blue gehört demnach einem vergangenen und inzwischen kritisierten Paradigma der KI-Forschung an.

Insbesondere die frühen Schriften von KI-Forschern hatten tatsächlich, wie auch schon anklang, die Ähnlichkeit von menschlicher und maschineller Informationsverarbeitung betont und ein tiefes Verständnis menschlicher Denkprozesse anvisiert. So erstaunt es nicht, dass die KI-Forschung immer wieder daran gemessen wird, ob das Computermodell des Geistes das menschliche Denken imitiert und zu dessen prinzipiellem Verständnis beiträgt.

Allerdings hatte bereits Alan Turing in seinem Aufsatz „Computing Machinery and Intelligence“ aus dem Jahr 1950 das Problem des maschinellen Denkens gewissermaßen auf pragmatischem Weg gelöst. Gleich zu Beginn seines Textes entzieht er sich bekanntermaßen einer Definition von Denken, die ihm fruchtlos erschien und ersetzte die Frage mit einem Spiel, dem Turing Test. Er nahm auch die Frage auf, ob dieser Test nicht zu einer reinen Simulation führen könne, als zu einer Form von „Intelligenz“, die dem menschlichen Denken nicht ähnelt. Turing schreibt: „Ist es nicht so, dass Maschinen etwas tun, das als Denken bezeichnet werden kann, das sich aber stark von dem unterscheidet, was Menschen tun?" Dieser Einwand sei schwerwiegend, so Turing, „doch können wir zumindest sagen, dass, auch wenn eine Maschine so gebaut werden kann, dass sie das Imitationsspiel zufriedenstellend spielt, wir dadurch nicht beunruhigt sein müssen" 
(Turing 2007 [1950]: 39). Nach Turing wäre in pragmatischer Weise vom maschinellen Denken zu sprechen, wenn die Maschine den Turing Test bestanden hat, also nicht mehr unterscheidbar von menschlicher Intelligenz ist, unabhängig davon, ob die Weise des Denkens die gleiche ist. ${ }^{21}$ Damit wurde aber bereits bei Turing von der Vorstellung Abstand genommen, dass die Computerintelligenz der menschlichen Intelligenz ähneln müsse. Vielmehr reicht es in der Logik des Turing Tests, wenn es nicht unterscheidbar ist. Im Hinblick auf das Computerschach gibt es im Übrigen immer wieder „Tests“, bei denen Schachspieler Spiele daraufhin beurteilen müssen, ob ein Computer oder ein Mensch spielt - was sie zumeist erkennen. Die Unterschiedlichkeit der „Denkweisen“ ist mithin sichtbar.

Auch McCarthy, Minsky, Rochester und Shannon hatten in ihrem Antrag für die Finanzierung der Dartmouth Conference im Jahr 1956 zwischen zwei Ansätzen unterschieden, um zu intelligenten Maschinen zu gelangen:

Two approaches, however, appear to be reasonable. One of these is to find how the brain manages to do this sort of thing and copy it. The other is to take some class of real problems, which require originality in their solution and attempt to find a way to write a program to solve them on an automatic calculator. Either of these approaches would probably eventually succeed. (McCarthy et al. 1955: 7)

Weiter hatten sie formuliert: „For the present purpose the artificial intelligence problem is taken to be that of making a machine behave in ways that would be called intelligent if a human were so behaving" (ebd.: 9). Mithin wird auch hier auf ein Verhalten rekurriert, das intelligent scheint. Ähnlich hatte Marvin Minksy später Künstliche Intelligenz definiert, indem er davon sprach, KI sei, wenn Maschinen Dinge tun, die, sofern sie von Menschen getan würden, einer Intelligenz bedürfen.

All diese Ausführungen verweisen weniger auf die Imitation des menschlichen Gehirns und dessen grundsätzliches Verständnis, sondern vielmehr darauf, mittels Computer Methoden zu entwickeln, mit denen Aufgaben übernommen werden, die vom Menschen ausgeübt, Intelligenz erfordern. Auch Shannon hatte in seinem Papier zum Computerschach die Unterschiede zwischen menschlicher und maschineller Intelligenz betont. Gerade die Typ A Strategie ahmt nicht die menschliche Weise des Schachspielens nach, wie in seinem Papier deutlich thematisiert wurde. Shannon schlägt jedoch folgende Strategie, gewissermaßen pragmatisch, bei der Erstellung eines Schachprogramms vor: „[it] should [match] to the capacities and weakness of the computer. The computer is strong in speed and accuracy and weak in analytical capabilities and recognition. Hence, 
it should make more use of brutal calculations than humans" (Shannon 1950: 273).

Zudem hatte Shannon, wie auch McCarthy im Hinblick auf die KIKonferenz in Dartmouth (u. a. McCarthy 1955), in seinem Aufsatz gleich zu Beginn betont, dass in der Forschung zum Schach nicht nur „theoretical possibilities“ lägen. Gleichzeitig hatte er potentielle Anwendungen genannt, die „worthy of serious considerations from the economic point of view“ (Shannon 1950: 256) seien. Unter anderem nannte er „Machines for performing symbolic (non-numerical) mathematical operations, Machines for making strategic decisions in simplified military operations, machines capable of orchestrating a melody; Machines capable of logical deduction“ (ebd.).

Norbert Wiener hatte Shannons Überlegungen aufgenommen und deutliche Bedenken, gerade hinsichtlich der Anwendung von KI, etwa in militärischen Kontexten, formuliert:

Shannon [...] zeigt unter anderem auch die Möglichkeit auf, daß ein solches Gerät der erste Schritt zur Konstruktion einer Maschine sein könnte, mit der man militärische Lagen auswerten und jeweils die beste Entscheidung treffen kann. Man denke nicht, daß er das leichthin sagt. [...] Wenn Shannon von der Entwicklung militärischer Taktiken spricht, redet er nicht ins Blaue hinein, sondern erörtert eine höchst aktuelle und bedrohliche Möglichkeit. (Wiener 1958: 174)

Wiener bezeichnete die mögliche Entwicklung einer KI als „klar und erschreckend“" (ebd.: 176).

Entscheidend an den Argumenten in den Publikationen der frühen KIForscher ist zweierlei. Erstens zeigt sich sehr deutlich, dass zwar die Nachahmung und das Verstehen des menschlichen Gehirns anhand von Computermodellen ein Ziel der KI war. Der Weg, das Gehirn mit Methoden zu simulieren, die gerade die Fähigkeiten des Computers im Unterschied zum menschlichen Gehirn (also Schnelligkeit, Genauigkeit, Fehlerlosigkeit) ausmachen, wurde allerdings von Beginn an gleichermaßen eingeschlagen - wie Shannon also formuliert hatte, war es ein Weg, gerade die Stärken der Maschine zu nutzen, über die Menschen nicht verfügten. So lässt sich festhalten, dass die KI von Anfang an, auf Anwendungen zielte und dabei auch den „Computerweg“ einschlug, der das Ziel grundlegender Ergebnisse über das menschliche Gehirn vernachlässigte. ${ }^{22}$

Mit dieser Perspektive auf die KI-Forschung stellt sich allerdings, zweitens, die Frage nach Erfolg oder Misserfolg bzw. „Sackgasse“ solcher Ergebnisse wie Deep Blue anders. Wenn es darum geht, menschliches Denken und Verhaltensweisen mit Computern lediglich zu simulieren, um Anwendungen hervorzubringen, kann Deep Blue als beachtlicher Erfolg gewertet 
werden (vgl. dazu auch Franchi \& Güzeldere 2005: $55 \mathrm{f}$.), auch wenn in heutigen Anwendungen der Konnektionismus und lernende Systeme eine weitaus größere Rolle spielen. Deep Blue steht dabei für den Erfolg der Anwendung, die auf nicht-menschlichem Wege erfolgt. Die Brute-ForceMethode ist für viele "search-based applications" von großer Bedeutung (Schaeffer \& van den Herik 2002: 6) und gerade heute in ihrem Erfolg offensichtlich. Auch IBM verfolgte mit der jahrelangen Entwicklung Deep Blues nicht vorrangig das Ziel, mit einem Schachcomputer zu beeindrucken und ein Medienspektakel zu inszenieren. Im Fokus standen konkrete und heute hochrelevante Anwendungen wie data mining, financial analysis (etwa Marktrends oder Risikoanalysen) und molecular modelling.

Dies verweist darüber hinaus auf zwei gänzlich unterschiedliche wissenschaftliche Konzepte: Einerseits auf eine auf grundlegende Prinzipien und Erklärungen setzende Wissenschaft, die gegen die vermeintlichen, aber doch „nur“ auf Computerpower basierenden Erfolge jeweils eine „wirkliche KI" einforderte, also fundamentale Erkenntnisse über die Prinzipien menschlichen Denkens. Dies basierte auf einem an menschlichen Denken, an Verstehen und Bewusstsein gebunden Begriff von Denken und Intelligenz. Andererseits verweist die Debatte um Deep Blue auf eine pragmatische Haltung, die auf die Simulation menschlichen Denkens und funktionierende Anwendung setzt, ohne dass die Methode des „Denkens“ der menschlichen Denkweise gleichen und ohne dass jedes Detail des Denkprozesses des Computers verstanden werden muss.

Dass die Brute-Force-Methode im Diskurs immer wieder so abgewertet wurde, hing, wie vor allem im nächsten Abschnitt zu sehen sein wird, einerseits mit der enttäuschten Erwartung zusammen, dass ein Computer auf gleiche Weise wie ein Mensch denken könne und beispielsweise zu einem „richtigen“ Verständnis von Schach gelangen würde. Es stand andererseits aber auch mit einer anthropozentrischen Verteidigungsstrategie des menschlichen, besonderen Denkens im Zusammenhang.

\section{Denken vs. stupides Rechnen: eine Grundsatzdebatte}

Die Debatte um die Frage des Denkens offenbarte diese enttäuschten Hoffnungen im Hinblick auf ein grundlegendes Verstehen menschlichen Denkens, die von den Kritikern gerade im Kontext der spektakulären und in den Medien gefeierten „Erfolge“ wie Deep Blue oder Watson umso deutlicher empfunden wurden. Im Grunde formulierte Kasparov nach seinem ersten Spiel gegen Deep Blue 1996 die entscheidende Frage: War es nun gerechtfertigt, von Denken zu sprechen oder nicht? 
Kasparov hatte das erste Spiel gegen Deep Blue 1996 im Nachhinein folgendermaßen kommentiert: „Zum erstenmal spürte, ja roch ich buchstäblich eine Art von Intelligenz auf der anderen Seite. Obwohl ich alles gab, spielte die Maschine ungerührt ein leichtes, wunderbares, fehlerfreies Schach. Ich war erschüttert. [...] Quantität schien in Qualität umzuschlagen. ${ }^{23}$ Auch hinsichtlich des zweiten Spiels im Turnier 1997 hatte Kasparov betont, dass es sich angefühlt habe, als hätte er gegen einen Menschen gespielt. Während er dies als Vorwurf, als Verdacht formulierte, es sei manipuliert worden, ${ }^{24}$ nahmen Vertreter der KI-Forschung wie Hans Moravec oder Ray Kurzweil, dies begeistert auf und interpretierten es als Beweis, dass Computer wie Menschen spielen könnten (Moravec 1998).

Monty Newborn drückte es folgendermaßen aus: „At the most fundamental level, Deep Blue's achievement provoked considerable thought on the subject of what intelligence is all about" (Newborn 2000: 27). Deep Blues Sieg warf insbesondere Fragen nach dem Verhältnis von Quantität und Qualität bzw. die Frage der Überführbarkeit von schierer Quantität in Qualität auf. Mehrfach finden sich Kommentare, die diese Frage thematisierten. ${ }^{25}$ Deep Blues Erfolg aktualisierte damit die seit einigen Dekaden geführte und die KI stets begleitende Debatte um die Frage, ob Maschinen denken können.

Vor allem Philosophen kritisierten das kognitivistische Paradigma, die Orientierung am Schach und den damit verbundenen Begriff von Intelligenz und Denken. ${ }^{26}$ Insbesondere Hubert Dreyfus und John Searles Kritik wurde vielfach rezipiert, auch innerhalb der KI-Forschung. Beide äußerten sich zu Deep Blue, dessen „Erfolg“ sie hinterfragten und mit ihren teils seit den 1960er Jahren erarbeiteten Argumenten gegen die KI-Forschung erneut kritisierten. Dabei argumentierten sie gleichermaßen gegen einen rein rationalen, formal-logischen Begriff des Denkens, wie er sich im 17. Jahrhundert herauszubilden begonnen hatte. So hatte Dreyfus bereits 1965 in seinem Aufsatz „Alchemy and Artificial Intelligence“ (Dreyfus 1965) eine sehr grundsätzliche Kritik an der KI-Forschung formuliert. In diesem Beitrag hatte er sich mit den Forschungsfeldern und dem Stand der KI auseinandergesetzt. Sein zentrales Argument war, dass es Kapazitäten und Eigenschaften des menschlichen Gehirns gäbe, die grundsätzlich nicht in Computer nachgebildet werden könnten. Er bezweifelte die Annahme der frühen KI-Forschung, dass das menschliche Gehirn in diskreten Operationen Informationen verarbeite. Es gäbe, so konstatierte er, keinen Beweis für die Annahme, dass Informationsverarbeitung im menschlichen Gehirn in gleicher Weise verlaufe wie im Computer. Er benannte insbesondere drei Eigenschaften des menschlichen Gehirns, die nicht im Computer nachbildbar seien. Im Hinblick auf das Schachspiel verwies er auf das Problem, aus der Vielzahl der möglichen Züge überhaupt die für den Spielverlauf rele- 
vanten Züge zu identifizieren - ein Problem, das, wie an Shannons Aufsatz von 1950 deutlich wird, auch innerhalb der KI-Forschung diskutiert wurde. Die exponentielle Zahl der Züge verunmögliche es, so argumentierte Dreyfus im Jahr 1965, dass der Computer dieses Problem mit brute force löse. Gleichzeitig sei die spezifische Fähigkeit der Menschen, sinnvolle Züge zu selektieren, nicht digital abbildbar. Im Grunde kam Dreyfus hier zu dem Schluss, dass die von Shannon entwickelte B-Strategie nicht formalisierbar und die A-Strategie mangels Computerpower nicht realisierbar sei. Er betrachtete die Fähigkeit des heuristischen Auswählens als ausschließlich menschliche Eigenschaft, die er in Anlehnung an William James das „fringe consciousness“ bezeichnete, ein „marginal“ oder „vague awareness“ (Dreyfus 1965: 21). Weiter nannte er als prinzipielle Grenzen der KI die Ambiguität der Sprache, die Tatsache, dass sich Sinn jeweils aus dem Kontext der Worte ergebe, weiter die Unfähigkeit von Computern, Wichtiges von Unwichtigem zu unterscheiden sowie drittens die hohe Komplexität der Welt, des menschlichen Alltags, die sich nur in einem leibhaften Umgang mit Welt erschließe, während Computer auf Mikrowelten beschränkt blieben. Der Kontrast des komplexen Alltags und der komplexen Fähigkeiten des menschlichen Gehirns zur geschlossenen, regelhaften Welt des Schachspiels wurde von ihm, und vielen anderen KI-Kritikern, stets betont.

In seinen späteren Publikationen entwickelte Dreyfus diese Argumente weiter, insbesondere in seinem in mehreren Auflagen erschienen Buch What Computer Can't Do (Dreyfus 1985). Auch in der Einleitung zur zweiten Auflage wiederholte er seine Argumente. In einer Auflage aus dem Jahr 1992 setzte er sich in einer weiteren Einleitung mit den neuronalen Netzen auseinander, die seine Grundskepsis jedoch nicht beseitigen konnten (Dreyfus 1992). Dreyfus hinterfragte mithin grundsätzlich die Grundannahmen der KI, insbesondere die Prämisse, Gehirn und Computer würden nach den gleichen Prinzipien funktionieren. Er bemängelte eine Verengung des Konzepts von Vernunft/Intelligenz auf rationales Denken, eine „platonische(n) Reduktion jeglichen Denkens auf explizite Regeln“ (Dreyfus 1985: 179). Intelligenz könne jedoch nicht mit „abstraktem logischem Denken“ gleichgesetzt werden (Dreyfus \& Dreyfus 1987: 17). Dreyfus kam daher zum Schluss, dass KI nie im menschlichen Sinne intelligent sein werde, auch nicht mit neuronalen Netzen.

Kurzum, im Kern seiner Aussagen steht eine grundsätzliche Skepsis, dass menschliches Denken mittels digitalem Computer nachgebildet werden könne. Ein „Erfolg“ wie Deep Blues Sieg sei keine Nachahmung menschlicher Intelligenz und bringe daher auch keine tiefen Einsichten und Erkenntnisse über menschliches Denken. Insbesondere in seinem Aufsatz von 1965 hatte Dreyfus betont, dass „there is no reason to deny 
the evidence that human and mechanical information processing proceed in entirely different ways" (Dreyfus 1965: 63). Allerdings gestand Dreyfus nach dem Sieg Deep Blues, überrascht gewesen zu sein. Nach dem Match fand in der US-amerikanischen Radiosendung NewsHour eine Diskussion zwischen Dreyfus und Daniel Dennett über die Relevanz des Sieges von Deep Blue für die KI-Forschung statt. Dieses Gespräch wurde in der elektronischen Zeitschrift Slate weitergeführt und 2005 nachgedruckt (vgl. Dreyfus \& Dennett 2005). Dreyfus insistierte auch hier, dass es Deep Blue nicht gelungen sei, Schach im menschlichen Sinne zu spielen. Mit Dennett stimmte er darin überein, dass Deep Blue für die KI-Forschung belanglos und die GOFAI eine Sackgasse sei. Das Gespräch wandte sich in dieser Einigkeit schnell von diesem Thema ab. Die beiden Philosophen diskutieren vielmehr den Roboter Cog, wobei sich im Prinzip die gleichen Argumentationslinien zeigten, dieses Mal hinsichtlich der Frage, inwieweit es gelingen könne, Emotionen zu simulieren. Dreyfus zog erneut grundsätzlich die Möglichkeiten der KI in Zweifel und ging von einer prinzipiellen Grenze digitaler Möglichkeiten aus (vgl. ebd.). Auch äußerte er sich abermals skeptisch, dass mit Methoden des Computers ernstzunehmende Erfolge zu erzielen seien. Er sprach von „symbol-crunching computers that would never even approach the problem-solving abilities of human beings" (zitiert nach Bringsjord 1998).

Die Kritik des Philosophen John R. Searle zielte in ähnlicher Weise auf den Begriff des Denkens, des Verstehens und insbesondere auf die Kategorie der Bedeutung ab. Bekannt und immer wieder zitiert und diskutiert ist sein Gedankenexperiment des „Chinesischen Zimmers“, mit dem er veranschaulichte, dass Computer zwar scheinbar Sinn produzieren können, jedoch keine Bedeutung verstehen (Searle 1986). Searles Ziel war es zu zeigen, dass die Frage, ob Computer denken können, strikt verneint werden müsse. Er verwies auf die rein formale Regelbefolgung des Computers, die, so sein Argument, nur intelligent zu sein scheint, tatsächlich jedoch kein Verstehen ermöglicht: „Syntax allein reicht nicht hin für Semantik, und digitale Computer haben, als Computer, kraft Definition bloß eine Syntax" (ebd.: 33).

In diesem Sinne hatte sich Searle auch im Hinblick auf Deep Blue geäußert. Er hatte Kurzweils Buch The Age of Spiritual Machine besprochen und diesem vorgeworfen, er habe Deep Blue als Beleg für ein Beispiel von Computerintelligenz bezeichnet (Searle 1999). Searle insistierte dagegen, dass Deep Blue nicht intelligent sei, sondern schlichtweg ein "Zahlenfresser“. Die Maschine denke nicht, spiele kein Schach, sie simuliere nicht einmal das Verhalten eines Schachspielers. Deep Blue sei weder eine Nachbildung des Menschen noch bringe er Erkenntnisse über das menschliche Gehirn. Searle argumentierte ganz im Sinne seines Gedankenexperiments. Er warf 
Kurzweil in einer weiteren Replik vor, dessen Ausführungen würden „suffer from a persistent confusion between simulating a cognitive process and duplicating it, and even worse confusion between the observer-relative, in-the eye-of-the beholder-sense of concepts like intelligence, thinking etc., and the observer-independent intrinsic sense" (ebd.). Kurz und vereinfacht zusammengefasst, kritisierte er, Kurzweil verwechsele die Simulation von Denken mit wirklichem Denken. Searle verweist immer wieder vor allem auf die Unmöglichkeit des Übergangs von Syntax zu Semantik sowie die Unmöglichkeit, von Quantität, wie sie Deep Blue par excellence verkörpere, zu Qualität zu gelangen.

Philosophische Kritiker der KI, von denen hier zwei prominente Beispiele stellvertretend etwas ausführlicher betrachtet wurden, widersprachen mithin prinzipiell der Möglichkeit, das menschliche Denken und die menschliche Intelligenz mit dem Computer nachzubilden. Deep Blue galt diesen Kritikern als Symbol eines formal-logischen Ansatzes der KIForschung, der als reduktionistisch und rein rechnend kritisiert wurde. Wie Manuela Lenzen zusammenfasste: „Die zentrale Kritik am Computermodell des Geistes lautet daher, dass es als Programm zur Modellierung menschlicher Intelligenz eine Sackgasse ist. Es führt kein Weg vom Schachcomputer Deep Blue zu den typischen Leistungen allgemeiner menschlicher Intelligenz" (Lenzen 2002: 65). Deep Blue beherrschte nur eine einzige Aufgabe, das Schachspielen, und damit war er weit entfernt von den Fähigkeiten des komplexen menschlichen Gehirns und dies tat er zudem mit rein rechnender Methode. Aus dieser Perspektive war Deep Blue eindeutig ein ärgerlicher Pseudo-Erfolg, der im Grunde ein Scheitern war.

Ganz im Sinne der gerade dargestellten Argumentation der philosophischen Kritik wurde das Computerschach auch in deutschen Schachzeitschriften, in denen Computerschach seit den 1970er Jahren ein immer wieder diskutiertes Thema war, kommentiert. ${ }^{27}$ Schachspielen bestehe, so die Kritik, nicht, wie es im Computerschach angenommen werde, aus einer "Reihe von logisch-mathematischen Operationen“. Ein Schachspieler führe nicht "nur logische Operationen aus“, er spiele auch und benötige daher Eigenschaften wie „Vorstellungskraft, Kombinationsfähigkeit, Scharfsinn, Witz, Mut, Vorsicht“. Er empfinde zudem während des Spiels Gefühle, wie Angst, Freude oder Hoffnung. Das Schachspiel könne daher nicht auf „pures Denken“ im Sinne logischer und berechenbarer Operationen reduziert werden. Ein Computer könne allenfalls einen elementaren Bestandteil des Schachspielens nachahmen, nicht jedoch das „Schachspiel als spezifisches Ganzes“. ${ }^{28}$ Auch hier wurde gegen die Tradition des formal-logischen Denkbegriffs argumentiert und auf die Bedeutung von Emotionen, Intuitionen, Erfahrung etc. verwiesen, weshalb das, was der Computer tue, nicht als denken bezeichnet werden könne. 
Auch der Exweltmeister Boris Spasski hatte 1981 kritisiert, dass Schachprogramme ein wenig rechnen könnten, jedoch die Prinzipien des positionellen Spiels nicht berücksichtigten. Er zeigte sich skeptisch, dass ein Schachcomputer ,je fähig sein wird, ähnlich wie ein Großmeister analytisch vorzugehen". ${ }^{29}$ Nicht zuletzt findet sich häufig das Argument, dass dem Computer etwas wie Intuition fehle, ${ }^{30}$ dass spielen nicht gleich rechnen sei $^{31}$ und dass die materielle Bewertung einer Position, also das $\mathrm{Zu}$ sammenrechnen der vorhandenen Figuren und der ihnen rechnerisch zugewiesenen Priorität, nicht mit menschlichem Schach zu vergleichen sei. ${ }^{32}$ Auch die Auswahl von „sinnvollen“ Zügen durch menschliche Schachspieler, die auf Erfahrung beruhe und nicht quantifizierbar sei, wurde immer wieder betont. ${ }^{33}$ Hoimar von Ditfurth wurde in der Deutschen Schachzeitung mit dem Verweis auf die Unterschiedlichkeit von Schachcomputer und menschlichem Schachspieler zitiert: Die „wahrhaft übermenschliche Rechengeschwindigkeit“, die „haushoch überlegene Speicherfähigkeit“ ermöglichten eine Brute-Force-Methode, doch sei dies nicht als intelligent zu bezeichnen. ${ }^{34}$ Schließlich wurde kritisch beobachtet, dass Computer Züge machten, die scheinbar die besten seien, es jedoch nicht nachvollziehbar sei, warum sie diese Züge machten und warum dies die besten Züge seien. Computer könnten selbst keine Begründung liefern: „Computer weisen auf der Grundlage des vollständigen Prüfens den Weg zum zwangsläufigen Gewinn, ohne dass wir bisher in der Lage sind, eine Strategie als Form der Begründung anzugeben“. ${ }^{35}$ Diese Beobachtung ist hochrelevant und aus heutiger Sicht auch brisant. Sie verweist zum einen auf die erwähnten unterschiedlichen Vorstellungen von Wissenschaft. Hier wird von Schachspielern, und damit auch vom Computer, das Begründen von Zügen eingefordert, mithin ein reflexives Element, das im Kontrast steht zur pragmatischen Beobachtung, dass der Schachcomputer funktioniere und gute Züge mache. Zum anderen verweist dies auf eine Beobachtung, die von KIKritikern immer wieder angeführt wird: „Entscheidungen“, die von Computern getroffen werden, ohne dass Menschen diese noch nachvollziehen können.

Innerhalb der KI-Forschung wurde Deep Blue dagegen als Erfolg gefeiert. Gleichwohl wurde hier auch auf die Grenzen Deep Blues verwiesen. Marvin Minsky konstatierte beispielsweise: „Deep Blue kann zwar ein Schachspiel gewinnen, aber er weiß nicht, dass er reingehen muss, wenn es regnet“. ${ }^{36}$ Und auch Ray Kurzweil stellte fest: „Deep Blue kann nur eine einzige intelligente Ausgabe ausführen, ist ansonsten völlig beschränkt und verfügt über keinen Kontext" (Kurzweil 1999a: 149). Tatsächlich hatte Kurzweil, anders als Searle es ihm vorgeworfen hatte, in seinem Buch geschrieben, dass Computer noch dumm seien. Er hatte jedoch auf dem Vorteil ihrer Schnelligkeit verwiesen, die schließlich auch Kasparov besiegt 
hätte. Kurzweil unterstrich, zumindest in seiner Replik auf Searle, dass Deep Blue gerade ein Ansatz sei, „to examine the human and [contemporary] machine approaches to chess [...], because [they] illustrate a clear contrast. Human thinking follows a very different paradigm" (Kurzweil 1999b). Er spricht statt dessen von einem funktionalem Äquivalent von Intelligenz, ähnlich wie bei einem Hörgerät, das das Hören wiederherstelle. ${ }^{37}$

Während Philosophen genau dies kritisierten, dass es der KI-Forschung nicht gelänge, die menschliche Intelligenz nachzuahmen, sondern sie diese schlicht mit Rechengewalt substituiere, simuliere und auf einen begrenzten Funktionsbereich beschränke, also funktional ersetze, waren viele KIForscher viel stärker mit der Machbarkeit von „intelligenten Maschinen“ beschäftigt, ohne dabei überhaupt zu behaupten, es handele sich um Denken im menschlichen Sinne. Viele Forscher, oft auch computer scientists, wie die Entwickler von Deep Blue, hatten schlichtweg Anwendungen im Sinn und scherten sich nicht um die philosophische Frage, ob dies Denken sei. Entsprechend behaupteten sie auch keineswegs, dass Deep Blue im menschlichen Sinne intelligent sei. Schon Shannon hatte die Schwächen der so genannten A-Strategie betont: „Even with the improvements we have discussed the above strategy gives an impression of relying too much on ,brute force' calculations rather than on logical analysis of a position“" (Shannon 1950: 273). Viele KI-Forscher waren sich der Überlegenheit aufgrund von Rechengeschwindigkeit und Rechenkapazität, die mit einem völligen Unverständnis von Schach einherging, völlig bewusst (etwa Buttazzo 2001: 25). Wie Moravec zusammefasste:

Engineers who know the mechanism of advanced robots most intimately will be the last to admit they have real minds. From the inside, robots will indisputably be machines, acting according to mechanical principles, however elaborately large. Only on the outside, where they can be appreciated as a whole, will the impression of intelligence emerge. (Moravec 1998)

Monty Newborn stellte hinsichtlich der Frage des „Denkens“ lakonisch fest: „No answers here“. Weiter resümierte er: „Deep Blue managed to play chess at the level of the world's greatest mind in a way quite different than that imagined by prominent scientists of the 1950s and 1960s" (Newborn 2000: 28). Gleichwohl ist das Zeigen von Intelligenz für die KIForscher entscheidend, auch wenn es auf rechnendem Weg und mit Computerpower ermöglicht werde. Newborn prognostizierte, dass genau diese Computerfähigkeit in Zukunft für viele Anwendungen nützlich sein würde (ebd.: 28). 
Interessant ist schließlich die Reaktion auf den Sieg von Deep Blue in den deutschen Medien, die tendenziell den kritischen Positionen entspricht und, wie auch die Haltung der zitierten Philosophen, dezidiert anthropozentrische Perspektiven spiegelten. Der Sieg Deep Blues hatte, wie bereits mehrfach erwähnt, ein immenses Medienecho hervorgerufen. Dabei wurde immer wieder betont, dass Schach als ein Spiel galt, das Intelligenz erfordere. Die menschlichen Fähigkeiten lagen, so das Image von Schach, in der Originalität, im Einfallsreichtum, im Umgang mit Komplexität oder der „erhabenen Täuschung“, so Kasparov. ${ }^{38}$ Der Sieg Deep Blues führte zu einer Debatte, was das Schachspielen ausmache. ${ }^{39}$ Dabei lassen sich zwei Diskursstränge unterscheiden. Zum einen wurde das menschliche Spiel in klarer Abgrenzung vom Computerschach beschrieben und dabei die der Maschine überlegenen menschlichen Eigenschaften betont. Stets wurde auch hier hervorgehoben, dass Menschen Stellungen erkennen, Analogien herstellen, Absichten erahnen, aus Erfahrung schöpfen, über Vorsicht, Aggressivität und Siegeswillen verfügen. Schach, das wurde unterstrichen, ganz im Sinne der oben zitierten Schachzeitschriften, sei nicht nur ein logisch-mathematisches Spiel. Mustererkennung und Muster in immer wieder in neue Zusammenhänge zu bringen, zeichne Schach genauso aus wie „die wunderbare Fähigkeit der Ahnung“. ${ }^{0}$ Die Zuschreibungen zum menschlichen Spielstil implizieren durchgängig solche positiven Wertungen. Stets wird die menschliche Kreativität unterstrichen, auch das Spielen „aus dem Bauch heraus“. So formulierte der Schachweltmeister Kramnik in einem Interview: „Ich weiß manchmal aus dem Bauch heraus, wie ich ziehen muss. Ich kann es nicht erklären, auch nicht mathematisch beweisen, ich fühle es einfach - und mein Gefühl hat mich selten getäuscht." ${ }^{41}$

Der Computer wurde dagegen tendenziell als ahnungslos und dumm dargestellt. Er rechne, sei präzise, arbeite mit „purer Rechengeschwindigkeit“, mit „roher Gewalt“. Er „ahnt nicht“, er bewege sich nie „im Ungefähren, sondern immer nur zwischen Ja und Nein, Schwarz und Weiß, Null und Eins.“42 Oder, wie ein Artikel in der Zeit es ausdrückte: Die Computer gewännen „mit Hilfe des primitiven, dafür aberwitzig schnellen Rechnens" ${ }^{43}$ So wurde in deutschen Zeitungen bis in die ersten 2000er Jahre hinein die Dummheit des Computers betont, sein reines, gewaltsames Rechnen.

Ein anderer Diskursstrang, der sich in der medialen Verarbeitung des Spektakels um Deep Blue findet, zeichnet sich durch eine Abwertung des Schachspiels aus. Es war von einem „Reputationsverlust“ des Schachs die Rede. Viele bezweifelten nun, ob das Spiel tatsächlich etwas mit Intelligenz zu tun habe, bis hin zur Bemerkung, Kasparov sei wohl gar kein tiefer Denker. Ein weiteres Argumentationsmuster lag also in der Abwertung dessen, was der Computer geschafft hatte, bzw. folgte der Logik, dass 
wenn es der Computer schaffe, es sich dabei um keine außergewöhnliche menschliche Leistung handeln könne. Wie auch ein englisch sprachiger Beobachter feststellte, hatte die Überlegenheit der Schachcomputer „very serious implications for the game of chess, which lost a lot of prestige as a result". Zum Beispiel seien Sponsorengelder zurückgezogen worden mit dem Hinweis, "that chess was no longer a serious game." ${ }^{44}$

Letztere Reaktion ist innerhalb der Geschichte der KI-Forschung häufig $\mathrm{zu}$ finden. Sie wird auch als KI-Effekt bezeichnet. Sobald ein Erfolg erzielt worden ist, wird dieser abgewertet mit dem Hinweis, dass es sich um keine komplexe Angelegenheit beziehungsweise doch nicht um „wirkliche KI" handele. Beide Argumentationsmuster finden sich auch im Hinblick auf Deep Blue wieder. Dies verbleibt allerdings in einer unreflektiert anthropozentrischen Perspektive, die auf der Sonderstellung der Menschen und ihrer einzigartigen Intelligenzleistung beharrt, dabei allerdings die wesentlichen Fragen verkennt, die sich aus dem „Erfolg“ Deep Blues sowie Watsons und Alpha Go ergeben.

\section{Das nächste Spektakel und die gleichen Diskussionen: Watson}

Der Sieg des IBM Computers Watson, benannt nach dem Firmengründer Thomas Watson, in der amerikanischen Quizshow Jeopardy im Jahr 2011 war fast genauso spektakulär wie Deep Blues Sieg über Kasparov. Auch dies wurde medial inszeniert und kommentiert. Und auch Watson löste eine Debatte aus, ob es sich denn um „richtige“ künstliche Intelligenz handele. Watson wurde von IBM entwickelt, um unstrukturierte Daten zu analysieren. ${ }^{45}$ Der Computer verfügt über Datenbanken und ist ein lernendes System, das in der Lage ist, Fragen zu beantworten und relevante Informationen aus Dateien zu extrahieren. Watson durchsucht Millionen von Dokumenten und nutzt dabei einen Algorithmus, der die Qualität der möglichen Antworten mit Hilfe von Statistik bewertet, um sie dann zu ranken.

In der amerikanischen Spielshow Jeopardy, in der Watson gegen die stärksten Spieler antrat, geht es darum, Fragen zu beantworten bzw. genauer, $\mathrm{zu}$ einer gegebenen Aussage, die passende Frage $\mathrm{zu}$ formulieren. So wird zum Beispiel der Satz: „In 1899 the Senate ratified the Treaty of Paris, ending this war" vorgegeben. Die zu findende Antwort bzw. Frage lautet: „What is the Spanish-American War?" (Rojas 2012). Im Quiz sind zudem Fangfragen und uneindeutige Fragen zu beantworten. Watson stellt keine simple Fortsetzung der Brute-Force-Methode dar, sondern ein lernendes System. Auch ist Watson nicht auf ein Feld, wie das Schachspielen 
mit seinen festgelegten Regeln, beschränkt. Gleichwohl spielt die Rechnerkapazität und -geschwindigkeit eine entscheidende Rolle. Watson hat aber zweifellos eine bisherige Grenze der KI überschritten, indem der Computer beginnt, Uneindeutigkeiten $\mathrm{zu}$ verstehen und Kontexte $\mathrm{zu}$ interpretieren. In Ansätzen zeigt Watson die Fähigkeit, die Ambiguität der Sprache zu „verstehen“ oder besser gesagt, mit ihr umzugehen - etwas das Dreyfus allein menschlicher Intelligenz zugeschrieben hat. ${ }^{46}$ Dabei handelt es sich allerdings, das ist unbestritten, wie bei Deep Blue und dem Schach, nicht um ein „wirkliches Verstehen“. Vielmehr arbeitet Watson auf statistischer Basis. Wie Raúl Rojas feststellte, kann man „DeepQA“ (tiefes Fragen-Antworten) „als ein statistisches Ensemble von Experten verstehen, die ein numerisches Votum (ein Score) für jede Hypothese abgeben“ (Rojas 2012). Gleichwohl liefert er beeindruckende Ergebnisse. Watson gestaltete im Juni 2016 die komplette Ausgabe eines Hefts der britischen Zeitschrift Drum. „Er wählte Bilder aus, passte Texte an und gestaltete die Seiten. Tausend Exemplare der Ausgabe wurden gedruckt". ${ }^{47}$ Inzwischen nutzt beispielsweise Associated Press eine Software, die täglich Millionen automatisierte Finanz- und Sportberichte produziert. ${ }^{48}$

Watson löste eine Debatte aus, die der um Deep Blue auffällig ähnelt. Es ist offensichtlich, dass auch Watson keine Einsichten in die Grundfragen nach den Prinzipien menschlichen Denkens und Verstehens liefert. Er ist keine „richtige“ künstliche Intelligenz. Noam Chomsky konstatierte in einem Interview, es sei nicht beeindruckt von einem „bigger steamroller“, wie er in Anspielung auf den „steamroller“ Deep Blue polemisierte. ${ }^{49}$ Chomsky beklagte erneut, dass keine Erkenntnisse für die KI-Forschung erzeugt würden. Diese Art der Forschung sei auf dem falschen Weg, wie er scharf kritisierte, da sie rein statistisch-probabilistisch vorgehe, es aber nicht mehr um Erklären und das Erkennen von Prinzipien oder um fundamentale Einsichten gehe. ${ }^{50}$ Auch Douglas Hofstadter kritisierte dass „these [Watson oder Apple's Siri] have nothing to do with thinking machines. ${ }^{\text {"51 }}$

Und, wie schon bei Searles Kritik an Deep Blue, meldete sich auch hier Ray Kurzweil zu Wort und konstatierte: „I would say that Watson is clearly not yet ,strong $\mathrm{AI}^{\prime}$, but it is an important step in that direction. It is the clearest demonstration I've seen of computers handling the subtleties of language including metaphors, puns and jokes, something people had said would not be possible." ${ }^{\text {" }}$

Die Debatte kreist mithin um die gleichen Argumente wie etwa 20 Jahre zuvor, als Deep Blue einen Schachweltmeister besiegt hatte. Einerseits wird wiederum bemängelt, dass quantitative, statistische und probabilistische Verfahren keine grundlegenden Erkenntnisse zur Funktionsweise des menschlichen Gehirns liefern und hier keineswegs von „Denken“ die Rede sein könne. Andererseits wird auf praktische Erfolge und Anwendungen 
verwiesen, bis wiederum dahin, dass dies als Weg hin zu einer wirklichen Intelligenz sei. Damit wird erneut die prinzipielle Frage, ob menschliches Denken mittels eines Computers nachgebildet werden kann, berührt. Immer wieder dreht sich die Debatte darum, ob es „wirklich“ Denken sei, womit stets menschliches Denken gemeint ist.

Deutlich wird erneut auch, dass es sich um zwei unterschiedliche Konzepte von Wissenschaft handelt: Ein Konzept, das, so die Selbstbeschreibungen, auf tiefes Verstehen und das Erkennen von Prinzipien zielt, sowie ein anderes, in dem es um die Entwicklung von Anwendung geht und bei dem irrrelevant ist, wenn sich die Methode dabei signifikant vom menschlichen Denken unterscheidet oder nicht alle Schritte des Computers verstanden werden. Denn was hierbei zählt, ist das Ergebnis.

Douglas Hofstadter kritisierte die Entwicklung der KI zu einer „engineering science“, die von Unternehmen wie IBM oder Google finanziert wird und daher auf "product development" fokussiert sei. ${ }^{53}$ Zweifellos verändert das Engagement kommerzieller und finanzstarker Firmen wie Google oder IBM massiv die KI-Forschung. Gleichwohl sollte im Vorherigen klar geworden sein, dass das Narrativ, die Kommerzialisierung verunreinige eine an fundamentalen Prinzipien orientierten KI-Forschung, zu simpel gestrickt ist. Die Anwendungsorientierung und ein gewisser Pragmatismus in der Konzentration auf das Machbare waren in der KI-Forschung bereits von Beginn an zu beobachten. Schließlich hat sich gerade der Weg der KI-Forschung als erfolgreich erwiesen, der schon bei Shannon als eine Alternative aufgezeigt wurde, nämlich der Weg, der nicht die menschliche Gehirnstruktur imitiert und gerade die Besonderheit des Computers im Vergleich zum menschlichen Denken nutzt. Entgegen der üblichen Erzählung der Geschichte der KI-Forschung, die eine klare Unterscheidung zwischen starker und schwacher KI trifft, ist zu unterstreichen, dass diese Unterscheidung in dieser Schärfe nicht haltbar ist. Erstens wurde gleichzeitig die Frage nach grundlegenden Prinzipien und Anwendungen gestellt, zweitens erhoffte man sich von der Beantwortung konkreter Anwendungsfragen grundlegende Erkenntnisse und umgekehrt; drittens ging man in der Forschung pragmatisch vor, indem Machbares, nämlich das überschaubare Schachspiel, in den Mittelpunkt geriet. Die Erfolge sind denn auch im Bereich der Anwendungen zu verzeichnen, die durchgängig auf der Basis mathematischer Verfahren, des Rechnens, der Statistik, der Wahrscheinlichkeiten und der Korrelationen erzielt werden. Wichtig ist es daher auch - um ein weiteres Standardnarrativ der KI-Forschung zu differenzieren -, nicht nur einen Wandel vom Kognitivismus zum Konnektionismus zu beschreiben, sondern auf die grundlegenden Kontinuitäten hinzuweisen. Wie auch Sven Winter fomulierte: „Kognitivismus und Konnektionismus sind letztlich verschiedene, aber nicht grundsätzlich unver- 
trägliche Varianten des Computermodell [...], die mit unterschiedlichen Arten von Berechnungsprozessen und Repräsentationen arbeiten. Da sich ihre Stärken und Schwächen im Wesentlichen ergänzen, liegt es nahe, sie in sogenannten hybriden Architekturen zu vereinen" (Winter 2014: 40).54

Unterstrichen wird dies durch den Erfolg der von Google entwickelten Software Alpha Go, die den als weltbesten Go-Spieler bezeichneten Lee Sedol im März 2016 besiegte. Alpha Go basiert auf lernenden neuronalen Netzwerken und riesigen Datenmengen mit Go-Partien. Rechnerkapazität, Datenmengen und lernende Systeme verbuchten, wie schon fünf Jahre zuvor beim IBM-Computer Watson, einen Erfolg der KI, der gleichfalls nichts mit menschlichem Denken zu tun hat.

\section{Die Frage nach dem Denken neugestellt: Konsequenzen, nicht Prinzipien}

Das Konstatieren einer grundlegenden Kontinuität mathematisch-statistischer Modelle sowie die Kontinuität einer engen Verflechtung und Gleichzeitigkeit von Interesse an theoretischen Einsichten und Anwendungen sowie die Erfolge der Anwendungen verweisen erstens darauf, dass die vorliegenden Überblicke zur KI-Forschung ergänzt und differenziert werden müssten. Bislang wird die Geschichte der KI zumeist als Paradigmenwechsel geschrieben. Notwendig wäre jedoch eine Geschichtsschreibung, die die KI in ihrer Wechselwirkung mit Gesellschaft und Ökonomie sowie insbesondere die Geschichte der Arbeitsteilung und Kooperation von Mensch und Computer, die aus ihren Anwendungen erfolgen, analysiert. Um dies an der Story um Deep Blue zu exemplifizieren: Der Einsatz des Computers veränderte das Schachspiel nachhaltig, ob als Konkurrent, als Trainingspartner oder mit der Verfügbarkeit von Informationen in Schachdatenbanken oder als Instrument, um eigene Schachpartien analysieren und alternative Züge anzeigen zu lassen. Der Computer wurde vom Konkurrent zum Partner, mit dem das Schachspiel sowie der/die menschliche Schachspieler/in ein/e andere/r wurde. ${ }^{55}$ Die genaue Analyse solcher Veränderungen steht nicht nur für das Computerschach aus, sondern für viele Felder.

Zweitens erscheint die Debatte, ob es sich jeweils um eine „wirkliche“ KI handele, ob Maschinen „wirklich“ denken und verstehen können, scholastisch und anthropozentrisch. Angesichts der Anwendungen der KI wäre eine weitaus relevantere Frage systematisch zu stellen, nämlich die, welche Konsequenzen es hat, wenn maschinelle Intelligenz, die gänzlich anders funktioniert und teils die Grenzen der menschlichen Verstehbarkeit über- 
schreitet, Aufgaben übernimmt, die zuvor von menschlicher Intelligenz ausgeführt wurden. Wie David Gelernter hinsichtlich Deep Blues Sieg formuliert hatte: „This match is almost a demonstration that intelligence is not the only way to win at chess [...]. Brute force, sheer computational mass, is something that can substitute for intelligence." ${ }^{\text {" } 6}$

Eine Frage, mit der sich auch Kasparov auseinandersetzte:

Yes, I think, we can hardly call it intelligence because we always believe that intelligence is something similar to our mind. But playing with Deep Blue, and other computers but mainly with Deep Blue, I can smell that the decisions that it's making are intelligent because I would come to the same conclusion by using my intuition. But if I use $90 \%$ of my intuition and positional judgement and $10 \%$ of calculation, and Deep Blue uses $95 \%$ of computation and $5 \%$ of built-in chess knowledge, and the result matches four times out of five, maybe we should talk about some sort of AI. ${ }^{57}$

Kasparovs Argument ist zweischneidig. Einerseits behält er den Begriff der „intelligence“ den Menschen vor, andererseits beobachtet er, dass es genauso aussehe, obwohl das Ergebnis auf gänzlich anderem Wege zustande kommt. Die entscheidende Frage, die sich anschließt, ist daher nicht mehr jene, die Dreyfus 1972 gestellt hatte - What Computers Can't Do bzw. ob das, was Computer tun, als Denken bezeichnet werden kann. Die entscheidende Aufgabe einer geistes- oder kulturwissenschaftlichen Forschung liegt vielmehr darin, die Unterschiede maschinellen und menschlichen Denkens genau zu beschreiben. Der Turing Test ist ja gerade deshalb noch nicht bestanden, weil sich die maschinelle Logik von der menschlichen Logik fundamental unterscheidet. Und selbst wenn es auf einer Beobachtungsebene nicht mehr zu unterscheiden wäre, wäre es umso wichtiger, die unterschiedlichen Denkweisen von Mensch und Computer, die unterschiedlichen Wege mit denen ein scheinbar gleiches Ergebnis erzielt wird, $\mathrm{zu}$ benennen. Dabei kann es nicht um eine essentialistische Aufrechterhaltung polarer Begriffe handeln. Anstelle anthropozentrischer Grenzziehungen, die den Begriff des Denkens Menschen vorbehalten wollen, ist es an der Zeit, das Tun der Maschinen als eine andere Form des Denkens zu beschrieben, ohne dabei allerdings wiederum in eine affirmative Computerbegeisterung zu verfallen. Der Fokus muss auf der Analyse der Unterschiede zum menschlichen Denken liegen, darauf, wie sich das Zusammenwirken und die enge Verflochtenheit von maschinellem und menschlichem Denken vollziehen und welche gesellschaftlichen Konsequenzen dies zeitigt. Es geht mithin nicht um die Stabilisierung von Mensch-MaschinenUnterschieden im anthropozentrischen Sinne, sondern um die Frage der Konsequenzen von Unterschieden. Ein zentraler Punkt ist hierbei, dass 
sowohl für das Computerschach als auch für Alpha Go konstatiert wurde, dass Computer Züge vornehmen, die von Menschen nicht verstanden werden. Hinsichtlich des Go-Spieles stellte die Profi-Spielerin Young Sun Yoon fest, Go habe Züge gemacht, „die ein Mensch nie machen würde. “58 Züge, die von den Beobachtern zuerst für einen Fehler gehalten wurden, schließlich aber zum Sieg führten. Anzuknüpfen ist daher an Joseph Weizenbaums Plädoyer, genau zu reflektieren, welche Aufgaben den auf ihre Maschinenweise denkenden Computern überlassen werden sollen (Weizenbaum 1978).

\section{Anmerkungen}

1 Game Over. Kasparov and The Machine, 2003; Computer Chess, 2013; Schach dem Roboter, Frankreich 1976.

2 Deutsche Schachzeitung, 130. Jg., Nr. 12, 1981, S. 410 f.

3 Deutsche Schachzeitung, 128. Jg., Nr. 3, 1979, S. 105.

4 Allerdings hatte er 1984 gegen Chess 4.7. ein Spiel verloren.

5 Schach im SPIEGEL, in: Der Spiegel, 13/1979, S. 3.

6 Die Erforschung des Wandels menschlicher Selbstbeschreibungen im Verhältnis zu Technik sowie sich verändernder Handlungen, Praktiken, Wahrnehmungen etc. ist ein Kernanliegen einer Historischen Technikanthropologie, die ich in anderen Beiträgen zu entwickeln versuche. In diesem Beitrag liegt der Fokus allerdings auf einer anderen Fragestellung.

7 Die Ansätze der embodied intelligence und die behaviour basierten Ansätze spielen im Folgenden keine Rolle.

8 Innerhalb der KI-Forschung wird zwischen starker und schwacher KI unterschieden. Während die schwache KI auf begrenzte Anwendungsfelder, etwa das Schachspielen, Expertensysteme, Übersetzungsprogramme etc., orientiert ist, zielt die sogenannte starke KI auf das Verständnis und die Nachbildung des komplexen menschlichen Gehirns.

9 Eine Geschichte des Computerschachs ist bislang nicht geschrieben.

10 Weitere Quellen sind Schachzeitschriften sowie Zeitungsartikel, insbesondere aus der Wochenzeitung Die Zeit, dem Magazin Der Spiegel und der Frankfurter Allgemeinen Zeitung.

11 Die Geschichte, der Ablauf des Turniers sowie die einzelnen Spiele und die entscheidenden Züge können im Detail etwa bei Hsu (2002), Goodman \& Keene (1997) und Pandolfini 1997 nachgelesen werden.

12 Maik Grosskathöfer, Speck für den Knecht, in: Der Spiegel, 24/2001, S. 166.

13 Das Spektakel um Deep Blue ist für die Frage nach der Selbstdeutung von Menschen im Verhältnis zur Technik aufschlussreich. Dieser Aspekt wird in diesem Beitrag, wie eingangs erwähnt, aber hier nicht verfolgt.

14 Ein tabellarischer Überblick über wichtige Ereignisse in der Geschichte des Computerschachs findet sich bei Franchi \& Güzeldere (2005: 128 f.). In Levy \& Newborn (1991) findet sich ein Überblick über die Geschichte des Computerschachs, vgl. weiter Coy (1993).

15 Herbert Simon nahm diese Metapher 1973 auf; seit Ende der 1970er Jahre ist sie häufig in der Literatur zu finden. 
16 Ensmenger vernachlässigt diese historische Perspektive, sodass in seinem Beitrag der Eindruck entsteht, die Entscheidung für das Schach habe zum kognitivistischen Paradigma geführt.

17 Das Papier von Newell, Shaw und Simon behandelt die von Shannon aufgeworfene Frage der Alternativen von heuristischer, selektiver Suche vs. Geschwindigkeit gleichfalls und präferiert ersteres.

18 Vgl. die Literaturangaben bei Ensmenger (2012: 23).

19 Wichtig ist zu betonen, dass es Forschungsansätze gab, die nicht an Shannons A-Strategie, sondern die B-Strategie anschlossen. Dazu gehören beispielsweise die Arbeiten des früheren russischen Großmeisters Michail Botwinnik. Diese Versuche, die vom dominanten Pfad abzuweichen versuchten, wurden allerdings bislang in der historischen Forschung nicht beachtet. Vgl. beispielweise die wenigen Bemerkungen bei Pfleger \& Weiner (1986: 24).

20 So konstatierte auch der Wissenssoziologe Harry Collins, dass das Schachparadigma und der rationalistische Weg die KI-Forschung verengt und weg von den Versuchen geführt hätte, menschliche Intelligenz zu simulieren. Viele weitere Autoren könnten hier genannt werden (Collins 2010: 108).

21 Vgl. hierzu auch Coy, der darauf verweist, dass Turing hier „rein behavioristisch“ argumentiere (Coy 1993: 211).

22 Die KI könnte daher als ein typisches Beispiel für den so genannten Mode 2 zur Produktion wissenschaftlichen Wissens bezeichnet werden. Der Begriff bezeichnet die Annahme, dass die Trennung von Grundlagen- und angewandter Forschung nicht gezogen werden kann; vgl. (Nowotny et al. 2004).

23 Interview mit Kasparov, in: Der Spiegel 18/1997, S. 224.

24 Interview mit Kasparov, in: Chess News 4/5/2005.

25 Wie Newborn zusammenfasste: "Can intelligence be quantified like chess rating?" (Newborn 2000: 28). Auch Goodman und Keene kommentierten: „Although Kasparov went on to win the match, it was clear that human intelligence was being challenged by a machine that seemed to transmute quantity of calculation into apparent quality of thought" (vgl. Goodman \& Keene 1997: 8).

26 Die Begriffe werden in der verwendeten Literatur oft nicht systematisch unterschieden.

27 Interessanterweise ist die Kommentierung des Computerschachs in der Zeitschrift des DDR-Schachverbands über weite Strecken eine andere als die hier skizzierte. Es kann hier nicht um einen systematischen Vergleich der Bewertung und Einordnung des Computerschachs in der Bundesrepublik und der DDR gehen. Zudem wären amerikanische Schachzeitschriften durchzusehen, zumal der philosophische Diskurs zur KI stark USamerikanisch geprägt ist.

28 M. Geppert, Computertod durch Schach?, in: Deutsche Schachzeitung, 129. Jg., Nr. 2, 1980, S. $41 \mathrm{f}$.

29 Interview mit Boris Spasski, in: Deutsche Schachzeitung, 130. Jg., Nr. 6, 1981, S. 203.

30 H. Bouwmeester, Gespräch mit einem Achtzigjährigen: Max Euwe, Deutsche Schachzeitung, 130. Jg., Nr. 9, 1981, S. 303-308, hier S. 307. Vgl. auch: Ernst Bönsch, Zum Transfer schachlichen Denkens auf Zweikampfsportarten und Sportspiele (II), in: Schach, Nr. 3, 1985, S. 102-103, hier 102.

31 Deutsche Schachzeitung, 132 Jg., Nr. 1, 1983, S. 39.

32 Deutsche Schachzeitung, 130. Jg. Nr. 10, 1981, S. 340 f.

33 Bönsch 1985: 102.

34 Deutsche Schachzeitung, 134. Jg. Nr. 5, 1985, S. 153.

35 Deutsche Schachzeitung, 135. Jg., Nr. 8, 1986, S. 313-315; sowie: Roderich Strobel, Wissenschaftlicher Spezialdiskurs in Dresden (IV), in: Schach, Nr. 6, 1989, S. 10-11, hier S. 11 . 
36 Zitiert in: Kurzweil (1999a: 148). Dies verweist auf die Erwartung an die KI, sich in der Welt verhalten zu können und nicht nur ein Feld zu beherrschen.

37 Wolfgang Coy wies darauf hin, dass die Vertreter der These der funktionalen Äquivalenz in der Regel nicht nach den Unterschieden menschlicher und maschineller Intelligenz fragen; vgl. Coy (1993: 215).

38 Kasparov im Interview, in: Der Spiegel 18/1997, S. 224.

39 Die folgenden Aussagen beziehen sich auf Zeitungsartikel aus der ZEIT, dem Spiegel und der FAZ im Zeitraum von 1997-2000.

40 Kasparov im Interview, in: Der Spiegel 18/1997, S. 224.

41 Kramnik im Interview, in: Der Spiegel 40/2002, S. 146.

42 Kasparov im Interview, in: Der Spiegel 18/1997, S. 224.

43 Klaus Manhart, Schachweltmeister Garri Kasparov tritt zum zweiten Mal gegen den IBM-Schachcomputer Deep Blue an, in: DIE ZEIT, 25. April 1997.

44 Tim Harding, How Much Longer Can Man Match the Computer? The Kibitzer, Chess Café, 7. Dezember 2003.

45 Vgl. die Selbstdarstellung auf der Homepage: http://www.ibm.com/watson/what-iswatson.html? mkwid=\&cm_mmc=\&cm_mmca2 (22.12.2016).

46 Dreyfus würde sicher argumentieren, dass Watson diese Ambiguität nicht wirklich versteht.

47 Adrian Lobo, Der Computer bestimmt, was auf die Titelseite kommt, in: Frankfurter Allgemeine Zeitung, 3. Juli 2016.

48 Ebd.

49 Interview mit Noam Chomsky zu IBM's Watson Computer vom 31. Januar 2011. URL: http://framingbusiness.net/archives/1287 (22.12.2016).

50 Noam Chomsky on Where Artificial Intelligence Went Wrong. An Extended Conversation with the Legendary Linguist. Interview von Yarden Katz. The Atlantic, 1. November 2012. URL: http://www.theatlantic.com/technology/archive/2012/11/noam-chomskyon-where-artificial-intelligence-went-wrong/261637/ (22.12.2016).

51 Why Watson and Siri are not real AI. Interview mit Douglas Hofstadter von William Herkewitz, 10. Februar 2014, in: Popularmechanics. URL: http://www. popularmechanics.com/science/a3278/why-watson-and-siri-are-not-real-ai$16477207 /(22.12 .2016)$.

52 Interview mit Noam Chomsky zu IBM’s Watson Computer vom 31. Januar 2011. URL: http://framingbusiness.net/archives/1287 (22.12.2016).

53 Why Watson and Siri are not real; vgl. ebd.

54 Er betont weiter: „Zu einem wirklich radikalen Bruch mit dem Computermodell und der traditionellen Auffassung von Kognition kam es erst mit dem Dynamizismus [...] und situierten Ansätzen“ (ebd.).

55 Dies wird in einem in Vorbereitung befindlichem Aufsatz bearbeitet.

56 Zitiert nach Weber (1996).

57 Kasparov im Interview: in: Goodman \& Keene (1997: 50).

58 Brettspiel-Turnier: Software schlägt Go-Genie mit $4 \mathrm{zu}$ 1, Spiegel Online, Dienstag, 15.03.2016. URL: http://www.spiegel.de/netzwelt/gadgets/alphago-besiegt-lee-sedolmit-4-zu-1-a-1082388.html (22.12.2016). 


\section{Literatur}

Becker, Barbara 1992. Künstliche Intelligenz. Konzepte, Systeme, Verheißungen. Frankfurt am Main: Campus.

Bell, Alex G. 1978. The Machine Plays Chess? Oxford: Pergamon Press.

Böhme, Hartmut 2009. Full Speed - Slow Down. Ambivalenzen der Moderne. In: Vera King und Genigna Gerisch (Hg.). Zeitgewinn und Selbstverlust: Folgen und Grenzen der Beschleunigung. Frankfurt am Main: Campus: 63-84.

Bolter, J. David 1984. Turing's Man. Western Culture in the Computer Age. Chapel Hill: University of North Carolina Press.

Bringsjord, Selmer 1998. Chess Is too Easy. MIT, Technology Review, March/April: 21-28.

Bruns, Edmund 2000. Das Schachspiel als Phänomen der Kulturgeschichte des 19. und 20. Jahrhunderts. Hamburg/London: Lit.

Buttazzo, Giorgio 2001. Artificial Consciousness: Utopia or Real Possibility? Computer, IEEE (34/7): 24-30.

Campbell, Murray A., Hoane, Joseph und Feng-Hsiung Hsu 2002. Deep Blue. Artificial Intelligence (134): 57-83.

Chomsky, Noam on Where Artificial Intelligence Went Wrong. An Extended Conversation with the Legendary Linguist. Interview von Yarden Katz. The Atlantic, 1. November 2012. URL: http://www.theatlantic.com/technology/archive/2012/11/noam-chomskyon-where-artificial-intelligence-went-wrong/261637. (22.12.2016).

Collins, Harry 2010. Tacit and Explicit Knowledge. Chicago: University of Chicago Press.

Coy, Wolfgang 1993. Matt nach 1060 Rechenschritten! In: Georg Hartwagner, Stefan Iglhaut und Florian Rötzer (Hg.). Künstliche Spiele. München: Klaus Boer Verlag: 202-218.

Dittmann, Frank 2015. Schachbrett zum Fußballfeld. In: Klaus Fuchs-Kittowski und Rainer E. Zimmermann (Hg.). Kybernetik, Logik, Semiotik. Philosophische Sichtweisen. Tagung aus Anlass des 100. Geburtstages von Georg Klaus. Berlin: Trafo Wissenschaftsverlag: $235-247$.

Dreyfus, Hubert 1965. Alchemy and Artificial Intelligence, Paper, Rand Cooperation. URL: http://www.rand.org/content/dam/rand/pubs/papers/2006/P3244.pdf (22.12.2016).

Dreyfus, Hubert 1985 [1972]. Die Grenzen künstlicher Intelligenz. Was Computer nicht können. Königstein: Athenäum.

Dreyfus, Stuart E. und Hubert Dreyfus 1987. Künstliche Intelligenz. Von den Grenzen der Denkmaschine und dem Wert der Intuition. Reinbek bei Hamburg: Rowohlt.

Dreyfus, Hubert 1992. What Computers Still Can't Do. A Critique of Artificial Reason. Cambridge/London: MIT Press.

Dreyfus, Hubert und Daniel Dennett 2005. Did Deep Blue's Win over Kasparov Prove that Artificial Intelligence Has Succeeded? A Debate. In: Stefano Franchi und Güven Güzeldere (Hg.). Mechanical Bodies, Computational Minds. Artificial Intelligence from Automata to Cyborgs. Cambridge/London: MIT Press: 265-280.

Ensmenger, Nathan 2012. Is Chess the Drosophila of Artificial Intelligence? A Social History of an Algorithm. Social Studies of Science (42): 5-30.

Franchi, Stefano und Güven Güzeldere 2005. Machination of the Mind: Cybernetics and Artificial Intelligence from Automata to Cyborgs. In: Dies. (Hg.). Mechanical Bodies, Computational Minds. Artificial Intelligence from Automata to Cyborgs. Cambridge/ London: MIT Press: $15-149$.

Goodman, David und Raymond Keene 1997. Man versus Machine. Kasparov versus Deep Blue. Cambridge, Mass: H 3 Publication.

Haugeland, John 1987. Künstliche Intelligenz - Programmierte Vernunft? Hamburg/New York: Mc-Graw-Hill Book Company.

Hsu, Feng-Hsiung 2002. Behind Deep Blue. Building the Computer that Defeated the World Chess Champion. Princeton/Oxford: Princeton University Press.

Krämer, Sybille 1988. Symbolische Maschinen. Die Idee der Formalisierung in geschichtlichem Abriß. Darmstadt: Wissenschaftliche Buchgesellschaft.

Kurzweil, Ray 1999a. Homo S@piens. Düsseldorf: Econ (original: 1998. The Age of Spiritual Machines: When Computers Exceed Human Intelligence. New York: Viking Press). 
Kurzweil, Ray 1999b. 'I Married a Computer': An Exchange. The New York Review of Books (May 20th). URL: http://www.nybooks.com/articles/1999/05/20/i-marrieda-computer-an-exchange (22.12.2016).

Lenzen, Manuela 2002. Natürliche und künstliche Intelligenz. Einführung in die Kognitionswissenschaft. Frankfurt am Main: Campus.

Levy, David 1976. Chess and Computer. London: Batsford Limited.

Levy, David und Monty Newborn 1991. How Computers Play Chess. New York: Freeman \& Company.

McCarthy, John, Marvin L. Minsky, Nathaniel Rochester und Claude E. Shannona. 1955. A Proposal for the Dartmouth Summer Research Project on Artificial Intelligence. URL: http://www-formal.stanford.edu/jmc/history/dartmouth/dartmouth.html(22.12.2016).

Moravec, Hans 1998. When will Computer Hardware Match the Human Brain. Journal of Evolution and Technology (1). URL: http://www.jetpress.org/volume1/moravec.pdf (22.12.2016).

Newborn, Monty 2003. Deep Blue: An Artificial Intelligence Milestone. New York: Springer.

Newborn, Monty 2000. Deep Blue's Contribution to AI. Annals of Mathematics and Artificial Intelligence (28): 27-30.

Newell, Allen, J. Cliff Shaw und Herbert Simon 1958. Chess-Playing Programs and the Problem of Complexity. IBM Journal of Research and Development (4): 320-335.

Nocks, Lisa 2008. The Robot. The Life Story of a Technology. Baltimore: Johns Hopkins University Press.

Nowotny, Helga, Peter Scott und Michael Gibbons 2004. Wissenschaft neu denken. Wissen und Öffentlichkeit in einem Zeitalter der Ungewißheit. Weilerswist: Velbrück.

Pfleger, Helmut und Ossi Weiner 1986. Schachcomputer. Gegner und Freund. München: Nymphenburger Verlagshandlung.

Rojas, Raúl 2012. Warum „Watson“ ein Durchbruch ist. Telepolis 24.03.2012. URL: http:// www.heise.de/tp/artikel/36/36578/1.html (22.12.2016).

Schaeffer, Jonathan und H. Jaap van den Herik 2002. Games, Computers, and Artificial Intelligence. Artificial Intelligence (134): 1-7.

Searle, John 1986. Geist, Hirn und Wissenschaft - Die Reith Lectures 1984. Frankfurt am Main: Suhrkamp.

Searle, John, 1999. I Married a Computer. New York Review of Books. http://www.nybooks. com/articles/1999/04/08/i-married-a-computer/. Zugegriffen: 22.12.2016.

Shannon, Claude E. 1950. Programming a Computer for Playing Chess. Philosophical Magazine (7): 256-275.

Turing, Alan 2007 [1950]. Computermaschinerie und Intelligenz. In: Karin Bruns und Ramón Reichert (Hg.). Neue Medien. Texte zur digitalen Kultur und Kommunikation. Bielefeld: transcript: $37-64$.

Turing, Alan 1987 [1953]. Spielprogramme. In: Bernhard Dotzler und Friedrich Kittler (Hg.). Intelligence Service, Alan M. Turing, Schriften. Berlin: Brinkmann \& Bose: 117-145.

Weber, Bruce 1996. It's Man Over Machine as Chess Champion Beats Computer He Calls Tough Opponent. New York Times February 18th, 1996.

Wiener, Norbert 1958. Mensch und Menschmaschine. Berlin: Ullstein.

Weizenbaum, Joseph 1978. Die Macht der Computer und die Ohnmacht der Vernunft. Frankfurt am Main: Campus.

Winter, Sven 2014. Kognition. Stuttgart: Reclam.

Zimmerli, Walther und Stefan Wolf 1994. Einleitung. In: Dies. (Hg.). Künstliche Intelligenz. Philosophische Probleme. Stuttgart: Reclam: 5-37. 


\section{Martina Heßler}

Professur für Neuere Sozial-, Wirtschafts- und Technikgeschichte Helmut-Schmidt-Universität

Holstenhofweg 85

22043 Hamburg

Deutschland

mhessler@hsu-hh.de 